T. Suzuki

Nagaoya Math. J.

Vol. 73 (1979) 117-147

\title{
ON ZETA FUNCTIONS ASSOCIATED WITH QUADRATIC FORMS OF VARIABLE COEFFICIENTS
}

\author{
TOSHIAKI SUZUKI
}

\section{Introduction}

In 1938, C. L. Siegel studied zeta functions of indefinite quadratic forms $([6], c)$. On the other hand, M. Sato and T. Shintani constructed the general theory of zeta functions of one complex variable associated with prehomogeneous vector spaces in 1974 ([1]). Moreover T. Shintani studied several zeta functions of prehomogeneous vector spaces, especially, "Dirichlet series whose coefficients are class-numbers of integral binary cubic forms" ([3]) and "Zeta functions associated with the vector space of quadratic forms" ([2]).

In this paper, we study zeta functions associated with quadratic forms of variable coefficients" which are closely related to zeta functions (indefinite) quadratic forms (in Siegel's sense) and zeta functions associated with the vector space of quadratic forms (in Shintani's sense).

Let $U$ be vector space of real symmetric matrices $X$ of size $n$, and $W$ be the vector space of $n$-dimensional column vectors $v$. The group $G L(n ; R)$ acts on $U$ by $X \mapsto g X^{t} g=X\left[{ }^{t} g\right](g \in G L(n ; R))$. For any $X \in U$, the isotropy subgroup $G(X)=O(X)$ of $X$ in $G L(n ; R)$ acts canonically on $W$. It is easy to see that the pairs $(G L(n ; \boldsymbol{R}), U)$ and $\left(O(X) \times \boldsymbol{R}_{+}^{\times}, W\right)$ are real forms of prehomogeneous vector spaces ( $\boldsymbol{R}_{+}^{\times}$acts on $W$ by multiplication). Let $M$ and $N$ be lattices in $U$ and $W$, which are invariant under the actions of the groups $G L(n ; Z) \subset G L(n ; R)$ and $O(X)_{Z}=$ $O(X) \cap G L(n ; Z) \subset O(X)$, respectively. By [1] and [6], c), if $n \geq 4$ and $\operatorname{det} X=|X| \neq 0$, we can consider "zeta functions of quadratic forms $X$ "

$$
\eta_{j}(s, N, X)=\sum_{\substack{v \in N / \sigma^{\prime} \\ \operatorname{sgn} X[v]=j}} \mu_{X}(v)|X[v]|^{-s}, \quad(j= \pm 1),
$$

where $N / \sim$ is the set of $O(X)_{Z}$-equivalence classes in $N$ and $\mu_{X}(v)$ are

Received January 12, 1978.

Revised March 16, 1978. 
defined by the normalized measure $d g_{X}$ on $O(X)$ such that

$$
\begin{gathered}
\int_{G L(n ; \boldsymbol{R})} f(g) d g=\int_{G L(n ; \boldsymbol{R}) / O(X)}\left\|X\left[^{t} \dot{g}\right]\right\|^{-(n+1) / 2} d\left(X\left[^{s} \dot{g}\right]\right) \cdot \int_{O(X)} f(\dot{g} g) d g_{X}(g), \\
\left(d g=\|g\|^{-n} \prod_{1 \leq i, j \leq n} d g_{i j},\left(g=\left(g_{i j}\right)\right), f(g) \in L^{1}(G L(n ; \boldsymbol{R}))\right) .
\end{gathered}
$$

Our "zeta functions" are defined as follows:

$$
\begin{aligned}
\xi_{i j}\left(s_{1}, s_{2}, L\right)= & \sum_{\substack{X \in M / \tilde{M} \\
\operatorname{sgn} X=(i, n-i)}} \eta_{j}\left(s_{2}, N, X\right)\|X\|^{-s_{1}-1 / 2} \\
\bar{\xi}_{i j}\left(s_{1}, s_{2}, \bar{L}\right)= & \sum_{\substack{Y \in M^{*} / \tilde{\sim} \\
\operatorname{sgn} Y=(i, n-i)}} \eta_{j}\left(s_{2}, N, Y^{c}\right)\|Y\|^{-s_{1}-(n-1) / 2}, \\
& (0 \leq i \leq n, j= \pm 1),
\end{aligned}
$$

where $L=M \oplus N, \bar{L}=M^{*} \oplus N\left(M^{*}\right.$ is the dual lattice of $\left.M\right), M / \sim$ and $M^{*} / \sim$ are the sets of $G L(n ; Z)$-equivalence classes on $M$ and $M^{*}$, respectively. Here $Y^{c}$ is the cofactor matrix of $Y$ and $G L(n ; R)$ acts contragrediently on the dual lattice $M^{*}$ of $M$.

On the other hand, we can see that the zeta functions $\xi_{i j}\left(s_{1}, s_{2}, L\right)$ and $\bar{\xi}_{i j}\left(s_{1}, s_{2}, \bar{L}\right)$ are also defined as zeta functions of two complex variables associated with the following prehomogeneous vector space $(G, \rho, V)$ and $(G, \bar{\rho}, \bar{V})$, respectively. This will be done in Section 2 . We identify the vector space $U$ with its dual $U^{*}$ by $\operatorname{Tr} X Y\left(X \in U, Y \in U^{*}\right)$, and identify $W$ its dual $W^{*}$ by ${ }^{t} v u\left(v \in W, u \in W^{*}\right)$. Let $\rho, \bar{\rho}$ and $\rho^{*}$ be the represensentations of $G=G L(n ; \boldsymbol{R}) \times \boldsymbol{R}^{\times}$on the vector spaces $V=U \oplus W, \bar{V}=$ $U^{*} \oplus W$ and $V^{*}=U^{*} \oplus W^{*}$ defined by

$$
\begin{aligned}
& \rho(g, h)(X, v)=\left(X\left[{ }^{t} g\right],{ }^{t} g^{-1} v h\right), \\
& \bar{\rho}(g, h)(Y, v)=\left(Y\left[g^{-1}\right],{ }^{t} g^{-1} v h\right)
\end{aligned}
$$

and

$$
\rho^{*}(g, h)(Y, u)=\left(X\left[g^{-1}\right], g u h^{-1}\right),
$$

respectively, where $g \in G L(n ; R), h \in \boldsymbol{R}^{\times}, X \in U, Y \in U^{*}, v \in W$ and $u \in W^{*}$. It is easy to see that complexifications of the triples $(G, \rho, V),(G, \bar{\rho}, \bar{V})$ and $\left(G, \rho^{*}, V^{*}\right)$ are prehomogeneous vector spaces.

In Section 4, we prove the main results of this paper (Theorem 2), which assert that, for $n \geqq 4, \xi_{i j}\left(s_{1}, s_{2}, L\right), \bar{\xi}_{i j}\left(s_{1}, s_{2}, \bar{L}\right)$ and $\xi_{i j}\left(s_{1}, s_{2}, L^{*}\right)$ are absolutely convergent for sufficiently large $\operatorname{Re}\left(s_{1}\right)$ and $\operatorname{Re}\left(s_{2}\right)$, and have 
analytic continuations to meromorphic functions of $\left(s_{1}, s_{2}\right)$ in the whole plane $C^{2}$ satisfying the following functional equations:

$$
\begin{aligned}
\bar{\xi}_{i j}( & \left.\frac{n+1}{2}-s_{1}-s_{2}, s_{2}, \bar{L}\right) \\
= & v\left(M^{*}\right)^{-1}(2 \pi)^{-n s_{1}-s_{2}} \pi^{n(n-1) / 4} \cdot e\left[\frac{n s_{1}+s_{2}}{1}\right] \\
& \cdot \Gamma\left(s_{1}\right) \Gamma\left(s_{1}-\frac{1}{2}\right) \cdots \Gamma\left(s_{1}-\frac{n-2}{2}\right) \Gamma\left(s_{1}+s_{2}-\frac{n-1}{2}\right) \\
& \cdot \sum_{\substack{0 \leq k \leq n \\
\ell= \pm 1}} A_{k \ell, i j}\left(s_{1}, s_{2}\right) \xi_{k \ell}\left(s_{1}, s_{2}, L\right)
\end{aligned}
$$

and

$$
\begin{aligned}
\xi_{i j}\left(s_{1}, s_{2}, L^{*}\right)= & v\left(N^{*}\right)^{-1} \pi^{2 s_{2}-n / 2-1} \Gamma\left(\frac{n}{2}-s_{2}\right) \Gamma\left(1-s_{2}\right) \\
& \cdot \sum_{\ell= \pm 1} B_{\ell j}^{i}\left(s_{2}\right) \bar{\xi}_{i \ell}\left(s_{1}+s_{2}-\frac{n-1}{2}, \frac{n}{2}-s_{2}, \bar{L}\right), \\
& \quad(0 \leqq i \leqq n, j= \pm 1),
\end{aligned}
$$

(for details, see Section 2 and 4).

The functional equations of "zeta functions" are based on their integral expressions and functional equations of "local zeta functions (Section 1 and 3)".

We know that zeta functions $\eta_{j}(s, N, X)$ have simple poles at $s=n / 2$ and $s=1$ with respective residues

$$
\begin{aligned}
& v(N)^{-1} \int_{O(X) / O(X) Z} d g_{X}=v(N)^{-1} \mu(X) \\
& \left(v(N)=\int_{W / N} d v, d v=d v_{1} \cdots d v_{n}\right)
\end{aligned}
$$

and

$$
v(N)^{-1} \mu^{\prime}(X) \pi^{1-n / 2} \Gamma\left(\frac{n}{2}-1\right) \begin{cases}\sin \pi(n-i) / 2 & \text { when } j=+1 \\ \sin \pi i / 2 & \text { when } j=-1\end{cases}
$$

where $\mu^{\prime}(X)=\|X\|^{1 / 2} \zeta(n-2) \sum_{1 \leq i \leq k} \mu_{X}\left(v_{i}\right)$, and $\left\{v_{1}, \cdots, v_{k}\right\}$ is a complete system of representatives of $O(X)_{Z}$-equivalence primitive points on $\{v \in N: X[v]=0, v \neq 0\}$ (see [1], §2,4). So, we may expect that zeta functions $\xi_{i j}\left(s_{1}, s_{2}, L\right)$ have poles at $s_{2}=n / 2$ and $s_{2}=1$ with respective residues 


$$
v(N)^{-1} \xi_{i}\left(s_{1}+\frac{1}{2}, M\right)
$$

and

$$
\begin{aligned}
v(N)^{-1} \xi_{i}^{\prime}\left(s_{1}+\frac{1}{2}, M \oplus N^{*}\right) \pi^{1-n / 2} \Gamma\left(\frac{n}{2}-1\right) \\
\begin{cases}\sin \pi(n-i) / 2 & \text { when } j=+1 \\
\sin \pi i / 2 & \text { when } j=-1\end{cases}
\end{aligned}
$$

where

$$
\xi_{i}\left(s_{1}+\frac{1}{2}, M\right)=\sum_{\substack{x \in M / \widetilde{M} \\ \operatorname{sgn} X=(i, n-i)}} \mu(X)\|X\|^{-s_{1}-1 / 2}
$$

are "zeta functions associated with the vector space of quadratic forms" and

$$
\xi_{i}^{\prime}\left(s_{1}+\frac{1}{2}, M \oplus N^{*}\right)=\sum_{\substack{x \in M / \\ \operatorname{sgn} X=(i, \tilde{N}-i)}} \mu^{\prime}(X)\|X\|^{-s_{1}-1 / 2} .
$$

In Section 5, we define the zeta functions $\xi_{i}^{\prime}\left(s, M \oplus N^{*}\right)$ by a similar way to the case of zeta functions associated with prehomogeneous vector spaces, which are in accord with the above formal residues. Further we prove the convergence of $\xi_{i}^{\prime}\left(s, M \oplus N^{*}\right)$ 's. The above formal argument is justified, and we obtain functional equations of $\xi_{i}(s, M)$ and $\xi_{i}^{\prime}\left(s, M \oplus N^{*}\right)$ (Theorem 3 ) which are inherited from that of $\xi_{i j}\left(s_{1}, s_{2}, L\right)$ 's. It is easy to see that the (inherited) functional equation of $\xi_{i}(s, M)$ is accord with the result of [2].

The author would like to thank Prof. T. Kubota, Prof. T. Shintani and Dr. Y. Kitaoka for their helpful advices.

\section{Notation}

For symmetric matrix $X$ of size $n, n$-dimensional column vector $v$, and $g \in G L(n ; R)$, we use following symbols:

$X[v]={ }^{t} v X v, X\left[{ }^{t} g\right]=g X^{t} g,|X|=\operatorname{det} X$.

$X^{c}$ : the cofactor matrix of $X\left(|X| \cdot X^{-1}\right.$ when $\left.|X| \neq 0\right)$.

$\|X\|,|X[v]|$ : the absolute value of $|X|$ and $X[v]$, respectively.

$\operatorname{sgn} X[v]=X[v] /|X[v]|$ when $X[v]$ is not zero.

$\operatorname{sgn} X=(i, n-i)$ when $|X|$ is not zero.

( $i$ : the number of positive eigenvalues of $X$ ) 
$e[x]=\exp (2 \pi \sqrt{-1} x),(x \in C)$.

$\mathscr{S}(V)$ : the space of rapidly decreasing functions on the vector space $V$.

$\mathscr{C}_{0}^{\infty}(E)$ : the space of complex-valued smooth functions on the smooth manifold $E$ with compact support.

$\boldsymbol{Q}_{p}$ : the field of $p$-adic numbers.

$Z_{p}$ : the ring of $p$-adic integers in $\boldsymbol{Q}_{p}$.

$\boldsymbol{F}_{p}$ : the fitite field with $p$ elements.

$|a|_{p}$ : the normalized absolute value of $a \in \boldsymbol{Q}_{p}$.

$\sharp[S]$ : the cardinality of a set $S$.

For an algebraic set $A$ defined over $\boldsymbol{Q}$, we denote by $A^{(p)}$ the algebraic set defined over $F_{p}$ obtained from $A$ by reduction modulo $p$.

\section{Local zeta functions}

In this section, we define "local zeta functions" of the triplets ( $G$, $\rho, V),(G, \bar{\rho}, \bar{V})$ and $\left(G, \rho^{*}, V^{*}\right)$. We write the elements of $V$ by $x$ or $(X, v)$, where $V=U \oplus W$ and $X \in U, v \in W$. Similarly, we write $\bar{x}=$ $(Y, v)$ and $x^{*}=(Y, u)$ for the elements of $\bar{V}=U^{*} \oplus W$ and $V^{*}=U^{*} \oplus W^{*}$, respectively.

The singular sets $S, \bar{S}$ and $S^{*}$ of $(G, \rho, V),(G, \bar{\rho}, \bar{V})$ and $\left(G, \rho^{*}, V^{*}\right)$ are respectively defined as follows:

$$
\begin{aligned}
& S=P \cup R, \quad P=\{(X, v) \in V:|X|=0\}, \quad R=\{(X, v) \in V: X[v]=0\}, \\
& \bar{S}=\bar{P} \cup \bar{R}, \quad \bar{P}=\{(Y, v) \in \bar{V}:|Y|=0\}, \quad \bar{R}=\left\{(Y, v) \in \bar{V}: Y^{c}[v]=0\right\}
\end{aligned}
$$

and

$$
\begin{aligned}
& S^{*}=P^{*} \cup R^{*}, \quad P^{*}=\left\{(Y, u) \in V^{*}:|Y|=0\right\}, \\
& R^{*}=\left\{(Y, u) \in V^{*} ; Y[u]=0\right\} .
\end{aligned}
$$

Put $\chi_{1}(g)=|g|^{2}$ and $\chi_{2}(h)=h^{2}\left(g \in G L(n: R), h \in R^{\times}\right)$, then we have

$$
\begin{aligned}
\left|X\left[{ }^{t} g\right]\right| & =\chi_{1}(g)|X|, & X\left[{ }^{t} g\right]\left[{ }^{t} g^{-1} v h\right] & =\chi_{2}(h) X[v], \\
\left|Y\left[g^{-1}\right]\right| & =\chi_{1}(g)^{-1}|Y|, & Y\left[g^{-1}\right]^{c}\left[{ }^{t} g^{-1} v h\right] & =\chi_{1}(g)^{-1} \chi_{2}(h) Y^{c}[v]
\end{aligned}
$$

and

$$
Y\left[g^{-1}\right]\left[g u h^{-1}\right]=\chi_{2}(h)^{-1} Y[u] .
$$

We set, for $0 \leq i \leq n$ and $j= \pm 1$,

$$
\begin{aligned}
& V_{i j}=\{(X, v) \in V-S: \operatorname{sgn} X=(i, n-i), \operatorname{sgn} X[v]=j\}, \\
& \bar{V}_{i j}=\left\{(Y, v) \in \bar{V}-\bar{S}: \operatorname{sgn} Y=(i, n-i), \operatorname{sgn} Y^{c}[v]=j\right\}
\end{aligned}
$$


and

$$
V_{i j}^{*}=\left\{(Y, u) \in V^{*}-S^{*}: \operatorname{sgn} Y=(i, n-i), \operatorname{sgn} Y[u]=j\right\} .
$$

Then we have

$$
V-S=\bigcup_{\substack{0 \leq i \leq n \\ j= \pm 1}} V_{i j}, \quad \bar{V}-\bar{S}=\bigcup_{\substack{0 \leq i \leq n \\ j= \pm 1}} \bar{V}_{i j}
$$

and

$$
V^{*}-S^{*}=\bigcup_{\substack{0 \leq i \leq n \\ j= \pm 1}} V_{i j}^{*} \quad \text { (disjoint union), }
$$

which are the orbital decompositions of $V-S, \bar{V}-\bar{S}$ and $V^{*}-S^{*}$ by the group $G^{+}=G L(n ; \boldsymbol{R})^{+} \times \boldsymbol{R}^{\times}$, respectively. We normalize Euclidian measures $d x, d \bar{x}$ and $d x^{*}$ on $V, \bar{V}$ and $V^{*}$ by $d x=d X \cdot d v, d \bar{x}=d Y \cdot d v$ and $d x^{*}=d Y \cdot d u$, respectively, where

$$
\begin{array}{llll}
d X=\prod_{1 \leq i \leq j \leq n} d x_{i j} & \left(X=\left(x_{i j}\right)\right), & d v=\prod_{1 \leq i \leq n} d v_{i} & \left(v=\left(v_{i}\right)\right), \\
d Y=\prod_{1 \leq i \leq j \leq n} d y_{i j} & \left(Y=\left(y_{i j}\right)\right), & d u=\prod_{1 \leq i \leq n} d u_{i} & \left(u=\left(u_{i}\right)\right) .
\end{array}
$$

For any rapidly decreasing functions $f(x) \in \mathscr{S}(V), \bar{f}(\bar{x}) \in \mathscr{S}(\bar{V})$ and $f^{*}\left(x^{*}\right) \in \mathscr{S}\left(V^{*}\right)$, we set

$$
\begin{aligned}
& \Phi_{i j}\left(f, s_{1}, s_{2}\right)=\int_{V_{i j}} f(x)\|X\|^{s_{1}}|X[v]|^{s_{2}} d x, \\
& \bar{\Phi}_{i j}\left(\bar{f}, s_{1}, s_{2}\right)=\int_{\bar{V}_{i j}} \bar{f}(\bar{x})\|Y\|^{s_{1}}\left|Y^{c}[v]\right|^{s_{2}} d \bar{x}
\end{aligned}
$$

and

$$
\Phi_{i j}^{*}\left(f^{*}, s_{1}, s_{2}\right)=\int_{V_{i j}^{*}} f^{*}\left(x^{*}\right)\|Y\|^{s_{1}}|Y[u]|^{s_{2}} d x^{*}
$$

$\left(\left(s_{1}, s_{2}\right) \in C^{2}, \operatorname{Re}\left(s_{1}\right)>0, \operatorname{Re}\left(s_{2}\right)>0,0 \leq i \leq n, j= \pm 1\right)$, respectively. It is clear that $\Phi_{i j}\left(f, s_{1}, s_{2}\right), \bar{\Phi}_{i j}\left(\bar{f}, s_{1}, s_{2}\right)$ and $\Phi_{i j}^{*}\left(f^{*}, s_{1}, s_{2}\right)$ are holomorphic functions of $\left(s_{1}, s_{2}\right) \in C^{2}$ in the region $\left\{\left(s_{1}, s_{2}\right) \in C^{2}: \operatorname{Re}\left(s_{1}\right)>0, \operatorname{Re}\left(s_{2}\right)>0\right\}$, and the mappings

$$
f(x) \longmapsto \Phi_{i j}\left(f, s_{1}, s_{2}\right), \quad \bar{f}(\bar{x}) \longmapsto \bar{\Phi}_{i j}\left(\bar{f}, s_{1}, s_{2}\right)
$$

and

$$
f^{*}\left(x^{*}\right) \longmapsto \Phi_{i j}^{*}\left(f^{*}, s_{1}, s_{2}\right)
$$


are tempered distributions on $V, \bar{V}$ and $V^{*}$. Now we call these distributions "local zeta functions" of $(G, \rho, V),(G, \bar{\rho}, \bar{V})$ and $\left(G, \rho^{*}, V^{*}\right)$, respectively.

THEOREM 1. (1) As analytic functions of $\left(s_{1}, s_{2}\right), \Phi_{i j}, \bar{\Phi}_{i j}$ and $\Phi_{i j}^{*}$ have analytic continuations in the whole complex plane $\boldsymbol{C}^{2}$.

(2) For any $\bar{f}(Y, v) \in \mathscr{S}(\bar{V})$, set

$$
f(X, v)=\int_{U^{*}} \bar{f}(Y, v) e[\operatorname{Tr} X Y] d Y
$$

Then we have $f(X, v) \in \mathscr{S}(V)$, and

$$
\begin{aligned}
& \Phi_{k \ell}\left(f, s_{1}-\frac{n}{2}, s_{2}-\frac{n}{2}\right)=(2 \pi)^{-n s_{1}-s_{2}} \pi^{n(n-1) / 4} \Gamma\left(s_{1}+s_{2}-\frac{n-1}{2}\right) \Gamma\left(s_{1}\right) \\
& \cdot \Gamma\left(s_{1}-\frac{1}{2}\right) \cdots \Gamma\left(s_{1}-\frac{n-2}{2}\right) e\left[\frac{n s_{1}+s_{2}}{4}\right] \\
& \quad \cdot \sum_{\substack{0 \leq i \leq n \\
j \leq \pm 1}} A_{k \ell, i j}\left(s_{1}, s_{2}\right) \bar{\Phi}_{i j}\left(\bar{f}, \frac{n-1}{2}-s_{1}-s_{2}, s_{2}-\frac{n}{2}\right), \\
& \quad(0 \leq k \leq n, \ell= \pm 1),
\end{aligned}
$$

where $A_{k \ell, i j}\left(s_{1}, s_{2}\right)$ are defined by

$$
\begin{aligned}
& A_{k \ell, i j}\left(s_{1}, s_{2}\right)=\left\{\begin{array}{l}
e\left[\frac{-(n+1) / 2+i}{4}\right] v_{k-1, i-1}^{(n-1)}\left(s_{1}\right) \\
e\left[-\frac{s_{1}+s_{2}-(n-1) / 2}{2}\right] e\left[\frac{(n+1) / 2-n+i}{4}\right] v_{k-1, i}^{(n-1)}\left(s_{1}\right) \\
e\left[-\frac{s_{1}+s_{2}-(n-1) / 2}{2}\right] e\left[\frac{(n+1) / 2-i}{4}\right] v_{k, i-1}^{(n-1)}\left(s_{1}\right)
\end{array}\right. \\
& e\left[\frac{-(n+1) / 2+n-i}{4}\right] v_{k, i}^{(n-1)}\left(s_{1}\right) \quad \text { when } \ell=+1, j=(-1)^{n-i+1}, \\
& w h e n l=-1, j=(-1)^{n-i+1},
\end{aligned}
$$




$$
(\sqrt{-1})^{-i(i+1) / 2} \alpha_{i r}= \begin{cases}(-1)^{(i-r) / 2}\left(\begin{array}{l}
i / 2 \\
r / 2
\end{array}\right) & \text { when } i \text { and } r \text { even, } \\
0 & \text { when } i \text { even, } r \text { odd }, \\
(-1)^{(i+1-r) / 2}\left(\begin{array}{c}
(i-1) / 2 \\
m / 2
\end{array}\right) & \text { when } i \text { odd, } r \text { even, } \\
(-1)^{(i-m) / 2}\left(\begin{array}{c}
(i-1) / 2 \\
(r-1) / 2
\end{array}\right) & \text { when } i \text { and } r \text { odd } .\end{cases}
$$

(3) For any $f^{*}(Y, u) \in \mathscr{S}\left(V^{*}\right)$, put

$$
\bar{f}(Y, v)=\int_{W^{*}} f^{*}(Y, u) e\left[{ }^{t} v u\right] d u .
$$

Then we have $\bar{f}(Y, v) \in \mathscr{S}(\bar{V})$ and

$$
\begin{gathered}
\bar{\Phi}_{k \ell}\left(\bar{f}, s_{1}+s_{2}-\frac{n+1}{2},-s_{2}\right)=\pi^{2 s_{2}-n / 2-1} \Gamma\left(\frac{n}{2}-s_{2}\right) \Gamma\left(1-s_{2}\right) \\
\quad \cdot \sum_{j= \pm 1} B_{\ell j}^{k}\left(s_{2}\right) \Phi_{k j}^{*}\left(f^{*}, s_{1}-\frac{n}{2}, s_{2}-\frac{n}{2}\right), \quad(0 \leq k \leq n, \quad \ell= \pm 1),
\end{gathered}
$$

where $B_{\ell j}^{k}\left(s_{2}\right)$ are defined by

$$
B_{\ell j}^{k}\left(s_{2}\right)= \begin{cases}-\sin \pi\left(k / 2-s_{2}\right), & \text { when } \ell=(-1)^{n-k}, j=+1, \\ \sin \pi k / 2, & \text { when } \ell=(-1)^{n-k}, j=-1, \\ \sin \pi(n-k) / 2, & \text { when } \ell=(-1)^{n-k+1}, j=+1, \\ -\sin \pi\left((n-k) / 2-s_{2}\right), & \text { when } \ell=(-1)^{n-k+1}, j=-1 .\end{cases}
$$

Let $|d / d Y|,(d / d Y)[v]$ and $Y[d / d v]$ be the differential operators on $\bar{V}$ defined by

$$
\begin{aligned}
& \left|\frac{d}{d Y}\right| e[\operatorname{Tr} X Y]=(2 \pi \sqrt{-1})^{n}|X| e[\operatorname{Tr} X Y], \\
& \frac{d}{d Y}[v] e[\operatorname{Tr} X Y]=2 \pi \sqrt{-1} X[v] e[\operatorname{Tr} X Y], \\
& \left.Y\left[\frac{d}{d v}\right] e\left[{ }^{t} v u\right]=(2 \pi \sqrt{-1})^{2} Y[u] e{ }^{t} v u\right],
\end{aligned}
$$

respectively. It is clear that $|d / d Y|(d / d Y)[v]=(d / d Y)[v]|d / d Y|$. Similarly we can define the differential operators $|d / d x|,(d / d x)^{c}[v]$ on $\bar{V}$ and $Y[d / d u]$ on $V^{*}$.

COROLlary to THEOREM 1. We have the following equations: 


$$
\begin{aligned}
\bar{\Phi}_{i j}\left(\left|\frac{d}{d Y}\right| \frac{d}{d Y}[v] \cdot \bar{f}, \frac{n-1}{2}-s_{1}-s_{2}, s_{2}-\frac{n}{2}\right) \\
=j\left(s_{1}+s_{2}-\frac{n-3}{2}\right)\left(s_{1}+s_{2}-\frac{n-1}{2}\right) s_{1}\left(s_{1}-\frac{1}{2}\right) \\
\quad \cdots\left(s_{1}-\frac{n-2}{2}\right) \bar{\Phi}_{i j}\left(\bar{f}, \frac{n-5}{2}-s_{1}-s_{2}, s_{2}-\frac{n}{2}+1\right) .
\end{aligned}
$$

(1-4) $\quad \Phi_{i j}\left(\left|\frac{d}{d X}\right|\left(\frac{d}{d X}\right)^{c}[u] \cdot f, s_{1}-\frac{n}{2}, s_{2}-\frac{n}{2}\right)$

$$
\begin{aligned}
= & j(-1)\left(s_{1}+s_{2}-\frac{n-3}{2}\right)\left(s_{1}-1\right)\left(s_{1}-1-\frac{1}{2}\right) \\
& \cdots\left(s_{1}-1-\frac{n-2}{2}\right) \cdot\left(s_{1}-2\right)\left(s_{1}-2-\frac{1}{2}\right) \\
& \cdots\left(s_{1}-2-\frac{n-2}{2}\right) \Phi_{i j}\left(f, s_{1}-\frac{n}{2}+2, s_{2}-\frac{n}{2}+1\right) .
\end{aligned}
$$

$(1-5) \quad \Phi_{i j}^{*}\left(Y^{c}\left[\frac{d}{d u}\right] \cdot f^{*}, s_{1}-\frac{n}{2}, s_{2}-\frac{n}{2}\right)$

$$
=4 j\left(s_{2}-1\right)\left(s_{2}-\frac{n}{2}\right) \Phi_{\imath \jmath}^{*}\left(f^{*}, s_{1}-\frac{n}{2}, s_{2}-\frac{n}{2}-1\right) \text {. }
$$

$$
\begin{aligned}
& \bar{\Phi}_{i j}\left(Y\left[\frac{d}{d v}\right] \bar{f}, s_{1}-s_{2}-1, s_{2}-\frac{n}{2}\right) \\
& \quad=4 j\left(s_{2}-1\right)\left(s_{2}-\frac{n}{2}\right) \bar{\Phi}_{i j}\left(\bar{f}, s_{1}-s_{2}-1, s_{2}-\frac{n}{2}-1\right) \\
& \quad(0 \leq i \leq n, j= \pm 1),
\end{aligned}
$$

Proof of Cor. In (1-1), we put $|d / d Y| \cdot(d / d Y)[v] \bar{f}$ instead of $\bar{f}$. Then $f$ is changed into $(-1)^{n+1}(2 \pi \sqrt{-1})^{n+1}|X| \cdot X[v] f$, and we have;

$$
\begin{aligned}
\Phi_{k \ell} & \left((-1)^{n+1}(2 \pi \sqrt{-1})^{n+1}|X| \cdot X[v] \cdot f, s_{1}-\frac{n}{2}, s_{2}-\frac{n}{2}\right) \\
& =(-2 \pi \sqrt{-1})^{n+1}(-1)^{n-k} \Phi_{k \ell}\left(f, s_{1}-\frac{n}{2}+1, s_{2}-\frac{n}{2}+1\right) .
\end{aligned}
$$

We calculate the above both members using (1-1) and the following equations

$$
A_{k \ell, i j}\left(s_{1}+1, s_{2}+1\right)=(-1)^{n-k} j \ell A_{k \ell, i j}\left(s_{1}, s_{2}\right) .
$$

Then we have (1-3). In order to obtain (1-4), we put $|Y| \cdot Y^{c}[v] \bar{f}$ instead of $\bar{f}$, then $f$ is changed into $|d / d X| \cdot(d / d X)^{c}[v] f(2 \pi \sqrt{-1})^{-2 n+1}$ and other parts are similar to the above. (q.e.d.) 
We consider differential forms $\omega_{0}, \bar{\omega}_{0}$ and $\omega_{0,}^{*}$ on $V-S, \bar{V}-\bar{S}$ and $V^{*}-S^{*}$, which satisfy the following equations:

$$
\begin{array}{ll}
d x=d(|X|) \wedge d(X[v]) \wedge \omega_{0} & \text { on } V-S, \\
d \bar{x}=d(|Y|) \wedge d\left(Y^{c}[v]\right) \wedge \bar{\omega}_{0} & \text { on } \bar{V}-\bar{S}
\end{array}
$$

and

$$
d x^{*}=d(|Y|) \wedge d(Y[u]) \wedge \omega_{0}^{*} \quad \text { on } V^{*}-S^{*},
$$

respectively, where $d x=d x_{11} \wedge d x_{12} \wedge \cdots \wedge d x_{n n} \wedge d v_{1} \wedge \cdots \wedge d v_{n}(x=$ $\left.(X, v), X=\left(x_{i j}\right), v=\left(v_{i}\right)\right)$ and so on. Next we define the differential forms $\omega, \bar{\omega}$ and $\omega^{*}$ on $V-S, \bar{V}-\bar{S}$ and $V^{*}-S^{*}$ by

$$
\begin{aligned}
& \omega=\|X\|^{-(n-2) / 2}|X[v]|^{-(n-2) / 2} \cdot \omega_{0}, \\
& \bar{\omega}=\left|Y^{c}[v]\right|^{-(n-2) / 2} \cdot \bar{\omega}_{0}
\end{aligned}
$$

and

$$
\omega^{*}=\|Y\|^{-(n-2) / 2}|Y[u]|^{-(n-2) / 2} \cdot \omega_{0}^{*},
$$

respectively. We may assume that $\omega_{0}, \bar{\omega}_{0}, \omega_{0}^{*}, \omega, \bar{\omega}$ and $\omega^{*}$ are all positive, because $V-S, \bar{V}-\bar{S}$ and $V^{*}-S^{*}$ are orientable. And we can identify the above differential forms with the measures on the following manifolds :

$$
\begin{aligned}
& V_{i j}\left(t_{1}, t_{2}\right)=\left\{x=(X, v) \in V_{i j}:\|X\|=t_{1},|X[v]|=t_{2}\right\} \\
& \bar{V}_{i j}\left(t_{1}, t_{2}\right)=\left\{\bar{x}=(Y, v) \in \bar{V}_{i j}:\|Y\|=t_{1},\left|Y^{c}[v]\right|=t_{2}\right\}
\end{aligned}
$$

and

$$
\begin{aligned}
V_{i j}^{*}\left(t_{1}, t_{2}\right)=\left\{x^{*}=(Y, u) \in\right. & \left.V_{i j}^{*}:\|Y\|=t_{1},|Y[u]|=t_{2}\right\}, \\
& \left(0 \leq i \leq n, j= \pm 1, t_{1}>0, t_{2}>0\right),
\end{aligned}
$$

respectively. For any $f(x) \in \mathscr{S}(V), \bar{f}(\bar{x}) \in \mathscr{S}(\bar{V})$ and $f^{*}\left(x^{*}\right) \in \mathscr{S}\left(V^{*}\right)$, set

$$
\begin{aligned}
& \Psi_{i j}\left(f, t_{1}, t_{2}\right)=\int_{V_{i j\left(t_{1}, t_{2}\right)}} f(x) \cdot \omega, \\
& \bar{\Psi}_{i j}\left(\bar{f}, t_{1}, t_{2}\right)=\int_{\bar{V}_{i j\left(t_{1}, t_{2}\right)}} \bar{f}(\bar{x}) \cdot \bar{\omega}
\end{aligned}
$$

and

$$
\begin{aligned}
& \Psi_{i j}^{*}\left(f^{*}, t_{1}, t_{2}\right)=\int_{V_{V_{j}^{*}\left(t_{1}, t_{2}\right)}} f^{*}\left(x^{*}\right) \omega^{*}, \\
&\left(0 \leq i \leq n, j= \pm 1, t_{1}>0, t_{2}>0\right),
\end{aligned}
$$


respectively. Then it is easy to see that

$$
\begin{aligned}
& \Psi_{i j}\left(f_{(g, h)}, t_{1}, t_{2}\right)=\Psi_{i j}\left(f, \chi_{1}(g) t_{1}, \chi_{2}(h) t_{2}\right), \\
& \bar{\Psi}_{i j}\left(\bar{f}_{(g, h)}, t_{1}, t_{2}\right)=\bar{\Psi}_{i j}\left(\bar{f}, \chi_{1}(g)^{-1} t_{1}, \chi_{1}(g)^{-1} \chi_{2}(h) t_{2}\right)
\end{aligned}
$$

and

$$
\Psi_{i j}^{*}\left(f_{(g, h)}^{*}, t_{1}, t_{2}\right)=\Psi_{i j}^{*}\left(f^{*}, \chi_{1}(g)^{-1} t_{1}, \chi_{2}(h)^{-1} t_{2}\right)
$$

where

$$
f_{(g, h)}(x)=f(\rho(g, h) x), \quad \bar{f}_{(g, h)}(\bar{x})=\bar{f}(\bar{\rho}(g, h) \bar{x})
$$

and

$$
f_{(g, h)}^{*}\left(x^{*}\right)=f^{*}\left(\rho^{*}(g, h) x^{*}\right)
$$

for $(g, h) \in G^{+}$. Moreover we have

$$
\begin{aligned}
\Phi_{i j}\left(f, s_{1}, s_{2}\right) & =\int_{0}^{\infty} \int_{0}^{\infty} t_{1}^{s_{1}+n / 2-1} t_{2}^{s_{2}+n / 2-1} \Psi_{i j}\left(f, t_{1}, t_{2}\right) d t_{1} d t_{2}, \\
\bar{\Phi}_{i j}\left(\bar{f}, s_{1}, s_{2}\right) & =\int_{0}^{\infty} \int_{0}^{\infty} t_{1}^{s_{1}+t_{2}+n / 2-1} \bar{\Psi}_{i j}\left(\bar{f}, t_{1}, t_{2}\right) d t_{1} d t_{2}
\end{aligned}
$$

and

$$
\begin{aligned}
\Phi_{i j}^{*}\left(f^{*}, s_{1}, s_{2}\right)= & \int_{0}^{\infty} \int_{0}^{\infty} t_{1}^{s_{1}+n / 2-1} t_{2}^{s_{2}+n / 2-1} \Psi_{i j}^{*}\left(f^{*}, t_{1}, t_{2}\right) d t_{1} d t_{2}, \\
& \left(0 \leq i \leq n, j= \pm 1, \operatorname{Re}\left(s_{1}\right)>0, \operatorname{Re}\left(s_{2}\right)>0\right) .
\end{aligned}
$$

\section{Zeta functions}

Set

$$
\begin{aligned}
G_{+}^{1} & =\left\{(g, h) \in G^{+}=G L(n ; R)^{+} \times R^{\times}: \chi_{1}(g)=1, \chi_{2}(h)=1\right\} \\
& =S L(n ; R) \times\{ \pm 1\} .
\end{aligned}
$$

We normalize the Haar measure $d G^{+}$on the group $G^{+}$by

$$
d G^{+}=|g|^{-n} \prod_{\substack{1 \leq i \leq n \\ 1 \leq j \leq n}} d g_{i j}|h|^{-1} d h, \quad\left(g=\left(g_{i j}\right) \in G L(n ; R)^{+}, h \in R^{\times}\right) .
$$

Then we can define the Haar measure $d^{1} g$ on the group $G_{+}^{1}$ by

$$
\begin{aligned}
\int_{G+} f(g, h) d G^{+}=\int_{G+/ G_{+}^{1}} \frac{d \chi_{1}(\dot{g})}{\chi_{1}(\dot{g})} \frac{d \chi_{2}(h)}{\chi_{2}(h)} \int_{G_{+}^{1}} f(\dot{g} g, h) d^{1} g, & \\
& \left(f(g, h) \in L_{1}\left(G^{+}\right)\right) .
\end{aligned}
$$

Set $G^{+}(x)=\left\{(g, h) \in G_{+}^{1}: \rho(g, h) x=x\right\}, \quad G^{+}(\bar{x})=\left\{(g, h) \in G_{+}^{1}: \bar{\rho}(g, h) \bar{x}=\bar{x}\right\}$ 
and $G^{+}\left(x^{*}\right)=\left\{(g, h) \in G_{+}^{1}: \rho^{*}(g, h) x^{*}=x^{*}\right\}$ for any $x \in V, \bar{x} \in \bar{V}$ and $x^{*} \in V^{*}$, respectively. Since $\|X\|^{-n / 2}|x[v]|^{-n / 2} d x,\|Y\|^{-1}\left|Y^{c}[v]\right|^{-n / 2} d \bar{x}$ and $\|Y\|^{-n / 2}$ $\cdot|Y[u]|^{-n / 2} d x^{*}$ are $G^{+}$-invariant measures on $V_{i j}, \bar{V}_{i j}$ and $V_{i j}^{*}$, respectively, there exist, for any $x \in V_{i j}, \bar{x} \in \bar{V}_{i j}$ and $x^{*} \in V_{i j}^{*}$, Haar measures $d \nu_{x}$, $d \nu_{\bar{x}}$ and $d \nu_{x^{*}}$ on the groups $G^{+}(x), G^{+}(\bar{x})$ and $G^{+}\left(x^{*}\right)$ such that

$$
\begin{aligned}
\int_{G^{+}} f(g, h) d G^{+}= & \int_{G^{+} / G^{+}(x)}\left\|X\left[{ }^{t} \dot{g}\right]\right\|^{-n / 2}\left\|X\left[{ }^{t} \dot{g}\right]\left[{ }^{t} \dot{g}^{-1} v h\right]\right\|^{-n / 2} \\
& \cdot d(\rho(\dot{g}, h) x) \cdot \int_{G^{+}(x)} f(\dot{g} g, h) d \nu_{x}(g), \\
\int_{G+} f(g, h) d G^{+}= & \int_{G^{+/ G+(\bar{x})}}\left\|Y\left[\dot{g}^{-1}\right]\right\|^{-1}\left|Y\left[\dot{g}^{-1}\right]^{c}\left[{ }^{t} \dot{g}^{-1} v h\right]\right|^{-n / 2} \\
& \cdot d(\bar{\rho}(\dot{g}, h) \bar{x}) \cdot \int_{G^{+}(\bar{x})} f(\dot{g} g, h) d \nu_{\bar{x}}(g)
\end{aligned}
$$

and

$$
\begin{aligned}
\int_{G^{+}} f(g, h) d G^{+}= & \int_{G+/ G+\left(x^{*}\right)}\left\|Y\left[\dot{g}^{-1}\right]\right\|^{-n / 2}\left|Y\left[\dot{g}^{-1}\right]\left[\dot{g} u h^{-1}\right]\right|^{-n / 2} \\
& \cdot d\left(\rho^{*}(\dot{g}, h) x^{*}\right) \cdot \int_{G^{+}\left(x^{*}\right)} f(\dot{g} g, h) d \nu_{x^{*}}(g), \\
& \left(f(g, h) \in L_{1}\left(G^{+}\right)\right),
\end{aligned}
$$

respectively. Now we can easily prove the following equations:

$$
\begin{gathered}
\int_{G_{+}^{1}} f_{1}(g) d^{1} g=\int_{G_{+}^{1} / G+(x)} \omega(\rho(\dot{g}, 1) x) \int_{G^{+}(x)} f_{1}(\dot{g} g) d \nu_{x}(g), \\
\int_{G_{+}^{1}} f_{1}(g) d^{1} g=\int_{G_{+}^{1} / G^{+}(\bar{x})} \omega(\bar{\rho}(\dot{g}, 1) \bar{x}) \int_{G^{+}(\bar{x})} f_{1}(\dot{g} g) d \nu_{\bar{x}}(g)
\end{gathered}
$$

and

$$
\begin{aligned}
\int_{G_{+}^{\mathrm{I}}}^{\mathrm{I}} f_{1}(g) d^{1} g=\int_{G_{+}^{1} / G+x^{*}} \omega\left(\rho^{*}(\dot{g}, 1) x^{*}\right) \int_{G^{+}\left(x^{*}\right)} f_{1}(\dot{g} g) d \nu_{x^{*}}(g), & \left(f_{1}(g) \in L_{1}\left(G_{+}^{1}\right)\right) .
\end{aligned}
$$

The complexifications of the triplets $(G, \rho, V),(G, \bar{\rho}, \bar{V})$ and $\left(G, \rho^{*}, V^{*}\right)$ have natural $\boldsymbol{Q}$-structures such that $G_{\boldsymbol{Q}}=G L(n ; \boldsymbol{Q}) \times \boldsymbol{Q}^{\times}, U_{\boldsymbol{Q}}$ is the set of rational symmetric matrices of size $n$, and $W_{Q}$ is the set of $n$-dimensional rational column vectors. Set $\Gamma=S L(n ; Z) \times\{ \pm 1\} \subset G_{+}^{1}$, and let $L=M \oplus N$ be a $: \Gamma$-invariant lattice in $V$, where $M$ and $N$ are lattices in $U$ and $W$, respectively. Write $M^{*}$ and $N^{*}$ for the dual lattices of $M$ and $N$, respectively, (i.e., $M^{*}=\left\{Y \in U^{*}: \operatorname{Tr} X Y \in Z\right.$ for any any $x \in M\}$ ). Then $\bar{L}=M^{*} \oplus N$ and $L^{*}=M^{*} \oplus N^{*}$ are also $\Gamma$-invari- 
ant lattices in $\bar{V}$ and $V^{*}$, respectively. We call two points in $V$ (or $\bar{V}$, $\left.V^{*}\right)$ " $\Gamma$-equivalent" if they lie in the same orbit of $\Gamma$. For any $\Gamma$ invariant subset $A$ of $V$ (or $\bar{V}, V^{*}$ ), let $A / \sim$ be the complete set of representatives of $\Gamma$-equivalence classes in $A$. We set

$$
L^{\prime}=L-S, \quad \bar{L}^{\prime}=\bar{L}-\bar{S}, \quad\left(L^{*}\right)=L^{*}-S^{*}
$$

and

$$
\begin{aligned}
L_{i j}=L \cap V_{i j}, \quad \bar{L}_{i j}=\bar{L} \cap \bar{V}_{i j}, \quad L_{i j}^{*}= & L^{*} \cap V_{i j}^{*}, \\
& (0 \leq i \leq n, j= \pm 1) .
\end{aligned}
$$

Now we define (formally) the following Dirichlet series which we call "zeta functions associated with quadratic forms of variable coefficients":

$$
\begin{aligned}
\bar{\xi}_{i j}\left(s_{1}, s_{2}, L\right) & =\sum_{x=(x, v) \in L_{i j} / \sim} \mu(x)\|X\|^{-s_{1}}|X[v]|^{-s_{2}}, \\
\bar{\xi}_{i j}\left(s_{1}, s_{2}, \bar{L}\right) & =\sum_{\bar{x}=(Y, v) \in \bar{L}_{i j} / \sim} \mu(\bar{x})\|Y\|^{-s_{1}}\left|Y^{c}[v]\right|^{-s_{2}}, \\
\xi_{i j}^{*}\left(s_{1}, s_{2}, L^{*}\right) & =\sum_{x^{*}=(Y, u) \in L_{i j}^{*} / \sim} \mu\left(x^{*}\right)\|Y\|^{-s_{1}}|Y[u]|^{-s_{2}}, \\
& \left(s_{1}, s_{2} \in C, 0 \leq i \leq n, j= \pm 1\right),
\end{aligned}
$$

where $\mu(x), \mu(\bar{x})$ and $\mu\left(x^{*}\right)$ are defined by

$$
\begin{aligned}
& \mu(x)=\int_{G+(x) / \Gamma_{x}} d \nu_{x}, \quad\left(\Gamma_{x}=\Gamma \cap G^{+}(x)\right), \\
& \mu(\bar{x})=\int_{G^{+}(\bar{x}) / \Gamma} d \nu_{\bar{x}}, \quad\left(\Gamma_{\bar{x}}=\Gamma \cap G^{+}(\bar{x})\right)
\end{aligned}
$$

and

$$
\mu\left(x^{*}\right)=\int_{G+\left(x^{*}\right) \Gamma x^{*}} d \nu_{x^{*}}, \quad\left(\Gamma_{x^{*}}=\Gamma \cap G^{+}\left(x^{*}\right)\right),
$$

respectively. It is easy to see that $\xi_{i j}^{*}\left(s_{1}, s_{2}, L\right)=\xi_{i j}\left(s_{1}, s_{2}, L\right)$, so that we write $\xi_{i j}\left(s_{1}, s_{2}, L^{*}\right)$ instead of $\xi_{i j}^{*}\left(s_{1}, s_{2}, L^{*}\right)$.

Then we can state the main results of this paper.

THEOREM 2. (1) For $n \geq 4$, there exist positive numbers $A$ and $B$ such that if $\operatorname{Re}\left(s_{1}\right)>A$ and $\operatorname{Re}\left(s_{2}\right)>B$ then $\xi_{i j}\left(s_{1}, s_{2}, L\right), \bar{\xi}_{i j}\left(s_{1}, s_{2}, \bar{L}\right)$ and $\xi_{i j}\left(s_{1}, s_{2}, L^{*}\right)$ are absolutely convergent, and they are analytic functions of $\left(s_{1}, s_{2}\right)$ in the region $\left\{\left(s_{1}, s_{2}\right) \in C^{2} ; \operatorname{Re}\left(s_{1}\right)>A, \operatorname{Re}\left(s_{2}\right)>B\right\}(0 \leq i \leq n$, $j= \pm 1)$.

(2) $\xi_{i j}\left(s_{1}, s_{2}, L\right), \bar{\xi}_{i j}\left(s_{1}, s_{2}, \bar{L}\right)$ and $\xi_{i j}\left(s_{1}, s_{2}, L^{*}\right)$ have analytic continua- 
tions to meromorphic functions of $\left(s_{1}, s_{2}\right)$ in the whole plane $C^{2}$ satisfying the following functional equations

$$
\begin{gathered}
\bar{\xi}_{i j}\left(\frac{n+1}{2}-s_{1}-s_{2}, \bar{L}\right)=v\left(M^{*}\right)^{-1}(2 \pi)^{-n s_{1}-s_{2}} \pi^{n(n-1) / 4} \\
\cdot e\left[\frac{n s_{1}+s_{2}}{4}\right] \Gamma\left(s_{1}\right) \Gamma\left(s_{1}-\frac{1}{2}\right) \cdots \Gamma\left(s_{1}-\frac{n-2}{2}\right) \\
\cdot \Gamma\left(s_{1}+s_{2}-\frac{n-1}{2}\right) \sum_{\substack{0 \leq k \leq n \\
\ell= \pm 1}} A_{k \ell, i j}\left(s_{1}, s_{2}\right) \xi_{k \ell}\left(s_{1}, s_{2}, L\right)
\end{gathered}
$$

$$
\begin{aligned}
& \xi_{i j}\left(s_{1}, s_{2}, L^{*}\right)=v\left(N^{*}\right)^{-1} \pi^{2 s_{2}-n / 2-1} \Gamma\left(\frac{n}{2}-s_{2}\right) \Gamma\left(1-s_{2}\right) \\
& \cdot \sum_{\ell= \pm 1} B_{\ell j}^{i}\left(s_{2}\right) \bar{\xi}_{i \ell}\left(s_{1}+s_{2}-\frac{n-1}{2}, \frac{n}{2}-s_{2}, \bar{L}\right) \\
& \quad(0 \leq i \leq n, j= \pm 1)
\end{aligned}
$$

where

$$
v\left(M^{*}\right)=\int_{U^{*} / M^{*}} d Y \quad \text { and } \quad v\left(N^{*}\right)=\int_{W^{*} / N} d u .
$$

(3) The functions

$$
\begin{aligned}
\left(s_{1}+\right. & \left.s_{2}-\frac{n-3}{2}\right)\left(s_{1}-1\right)\left(s_{1}-1-\frac{1}{2}\right) \cdots\left(s_{1}-1-\frac{n-2}{2}\right) \\
& \cdot\left(s_{1}-2\right)\left(s_{1}-2-\frac{1}{2}\right) \cdots\left(s_{1}-2-\frac{n-2}{2}\right)\left(s_{2}-1\right) \\
& \left(s_{2}-\frac{n}{2}\right) \xi_{i j}\left(s_{1}, s_{2}, L\right)
\end{aligned}
$$

and

$$
\begin{gathered}
\left(s_{1}-1\right)\left(s_{1}-2\right)\left(s_{1}+s_{2}-1-\frac{1}{2}\right)\left(s_{1}+s_{2}-1-\frac{2}{2}\right) \\
\ldots\left(s_{1}+s_{2}-1-\frac{n-1}{2}\right)\left(s_{2}-1\right)\left(s_{2}-\frac{n}{2}\right) \\
\cdot \bar{\xi}_{i j}\left(s_{1}, s_{2}, \bar{L}\right), \quad(0 \leqq i \leqq n, j= \pm 1),
\end{gathered}
$$

of $\left(s_{1}, s_{2}\right)$ are entire on the whole plane $C^{2}$.

\section{Proof of Theorem 1}

We introduce the following distributions for fixed $v_{0}={ }^{t}(1,0, \cdots, 0)$ $\in W$. For any $f(X) \in \mathscr{S}(U)$ and $f^{*}(Y) \in \mathscr{S}\left(U^{*}\right)$, we set

$$
\Omega_{i j}\left(f, s_{1}, s_{2}\right)=\int_{\substack{\operatorname{sgn} X=(i, n-i) \\ \operatorname{sgn} X\left[v_{0}\right]=j}} f(X)\|X\|^{s_{1}}\left|X\left[v_{0}\right]\right|^{s_{2}} d X,
$$




$$
\begin{aligned}
& \Omega_{i j}^{*}\left(f^{*}, s_{1}, s_{2}\right)=\int_{\substack{\operatorname{sgn} Y=(i, n-i) \\
\operatorname{sgn} Y\left[v_{0}\right]=j}} f^{*}(Y)\|Y\|^{s_{1}}\left|Y^{c}\left[v_{0}\right]\right|^{s_{2}} d Y, \\
&\left(s_{1}, s_{2} \in C, \operatorname{Re}\left(s_{1}\right)>0, \operatorname{Re}\left(s_{2}\right)>0,0 \leq i \leq n, j= \pm 1\right) .
\end{aligned}
$$

It is known that the mappings

$$
f(X) \longmapsto \Omega_{i j}\left(f, s_{1}, s_{2}\right), \quad f^{*}(Y) \longmapsto \Omega_{i \jmath}\left(f^{*}, s_{1}, s_{2}\right)
$$

are tempered distributions which have analytic continuations to meromorphic functions of $\left(s_{1}, s_{2}\right)$ in the whole plane $C^{2}$ (see [4]).

LEMMA 3-1. For any $f^{*}(Y) \in \mathscr{S}\left(U^{*}\right)$, if we set

$$
f(X)=\int_{U^{*}} f^{*}(Y) e[\operatorname{Tr} X Y] d Y,
$$

then $f(X) \in \mathscr{S}(U)$ and

$$
\begin{aligned}
& \Omega_{k \ell}\left(f, s_{1}-\frac{n}{2}, s_{2}-\frac{n}{2}\right)=(2 \pi)^{-n s_{1}-s_{2}} \pi^{n(n-1) / 4} e\left[\frac{n s_{1}+s_{2}}{4}\right] \\
& \cdot \Gamma\left(s_{1}\right) \Gamma\left(s_{1}-\frac{1}{2}\right) \cdots \Gamma\left(s_{1}-\frac{n-2}{2}\right) \Gamma\left(s_{1}+s_{2}-\frac{n-1}{2}\right) \\
& \cdot \sum_{\substack{0 \leq i \leq n \\
j= \pm 1}} A_{k \ell, i j}\left(s_{1}, s_{2}\right) \Omega_{i j}^{*}\left(f^{*}, \frac{n-1}{2}-s_{1}-s_{2}, s_{2}-\frac{n}{2}\right) \\
& \quad(0 \leq k \leq n, \ell= \pm 1) .
\end{aligned}
$$

Proof. This is a similar calculation to Lemma 4.1 of [1]. Also, we can derive the above result by the method of "microlocal calculus" (see [14]), too.

Remark. $v_{k, i}^{(n)}(s)$ 's are expressed by the following generating function.

$$
\begin{array}{r}
\sum_{0 \leq k \leq n} v_{k, i}^{(n)}(s) T^{k}=e\left[\frac{-n s}{4}\right] \prod_{1 \leq p \leq n-i}\left(e\left[\frac{s-(n+1) / 2+p}{4}\right]\right. \\
\left.+e\left[\frac{s-(n+1) / 2+p}{4}\right] T\right) \\
\cdot \prod_{1 \leq q \leq i}\left(e\left[-\frac{s-(n+1) / 2+q}{4}\right]+e\left[\frac{s-(n+1) / 4+q}{4}\right] T\right),
\end{array}
$$

where $T$ is indeterminant (see [2], [14]). It is easy to see that;

$$
\begin{aligned}
& \frac{e[s / 2] v_{k, i}^{(n)}(s)-v_{k, i-1}^{(n)}(s)}{e[s / 2]-e[-s / 2]}=e\left[\frac{-(n+1) / 2+i}{4}\right] v_{k-1, i-1}^{(n-1}\left(s-\frac{1}{2}\right), \\
& \frac{-e[-s / 2] v_{k, 2}^{(n)}(s)+v_{k, i+1}^{(n)}(s)}{e[s / 2]-e[-s / 2]}
\end{aligned}
$$




$$
\begin{gathered}
=e\left[-\frac{2 s-(n+1) / 2+n-i}{4}\right] v_{k-1, i}^{(n-1)}\left(s-\frac{1}{2}\right), \\
\frac{-e[-s / 2] v_{k, i}^{(n)}(s)+v_{k, i-1}^{(n)}(s)}{e[s / 2]-e[-s / 2]}=e\left[-\frac{2 s-(n+1) / 2+i}{4}\right] v_{k, i-1}^{(n-1)}\left(s-\frac{1}{2}\right), \\
\frac{e[s / 2] v_{k, i}^{(n)}(s)-v_{k, i+1}^{(n)}(s)}{e[s / 2]-e[-s / 2]}=e\left[\frac{-(n+1) / 2+n-i}{4}\right] v_{k, i}^{(n-1)}\left(s-\frac{1}{2}\right) .
\end{gathered}
$$

Proof of Theorem 1. In Lemma 3-1, we replace $v_{0}$ by arbitrary $v$ in $W$, then we can see that functional equations are unchanged. Replacing $f^{*}(Y)$ by $\bar{f}(Y, v) \in \mathscr{S}(\bar{V})$, we integrate those functional equations on parameter $v$, then we obtain (1) of Theorem 1 . In similar way, we obtain (2) of Theorem 1 by integrating "Formula of Fourier transforms of distributions attached to quadratic forms $Y[v]$ " ([5], [6]) on parameter $Y$.

Note. At first the author calculated the results (1) of Theorem 1 in a different way. The above simple method was noticed later. On the other hand, F. Sato, who were studying" Eisentein series for indefinite quadratic forms [13]" by a similar way to [1], pointed out the fact, too.

\section{Proof of Theorem 2.}

First, we prove the convergence of "zeta functions". It is easy to see that the following method is applicable to the cases of many prehomogeneous spaces.

Let $t_{1}$ and $t_{2}$ be non-zero rational numbers. We define

$$
V\left(t_{1}, t_{2}\right)=\left\{(X, v) \in V^{c}:|X|=t_{1}, X[v]=t_{2}\right\}
$$

and

$$
\bar{V}\left(t_{1}, t_{2}\right)=\left\{(Y, v) \in \bar{V}^{c}:|Y|=t_{1}, Y^{c}[v]=t_{2}\right\},
$$

which are affine varieties defined over $\boldsymbol{Q}$. We set

$$
\begin{array}{lr}
V\left(t_{1}, t_{2}\right)_{R}=V\left(t_{1}, t_{2}\right) \cap V, \quad V\left(t_{1}, t_{2}\right)_{Q}=V\left(t_{1}, t_{2}\right) \cap V_{Q}, \\
L\left(t_{1}, t_{2}\right)=V\left(t_{1}, t_{2}\right) \cap L, \quad L_{i j}\left(t_{1}, t_{2}\right)=V\left(t_{1}, t_{2}\right) \cap L_{i j}, \\
\quad(0 \leq i \leq n, j= \pm 1) .
\end{array}
$$

Similarly, set for $\bar{V}_{R}\left(t_{1}, t_{2}\right)$, etc. We show that, if $n \geq 4$, there exist positive numbers $A^{\prime}, B^{\prime}$ and $C$ such that

$$
\begin{aligned}
& \sum_{x \in L_{i j}\left(t_{1}, t_{2}\right) / \sim} \mu(x)<\left.C\left|t_{1}\right|\right|^{A^{\prime}}\left|t_{2}\right|^{B^{\prime}}, \\
& \sum_{\bar{x} \in \bar{L}_{i j}\left(t_{1}, t_{2}\right) / \sim} \mu(\bar{x})<C\left|t_{1}\right|^{A^{\prime}}\left|t_{2}\right|^{B^{\prime}}, \quad(0 \leqq i \leq n, j= \pm 1) .
\end{aligned}
$$


By Lemma 2-3 in [1], we have

$$
\int_{G_{+}^{1} / \Gamma} \sum_{x \in L^{\prime}} f(\rho(g, 1) x) d^{1} g=\sum_{x \in L^{\prime} / \sim} \mu(x) \int_{\rho\left(G_{+}^{1}\right) x} f \cdot \omega, \quad(f(x) \in \mathscr{S}(V)) .
$$

For the sake of brebity we set $g=(g, 1) \in G_{+}^{1}=S L(n ; R) \times\{ \pm 1\}$. We may assume that $f \geqq 0$ and the support of $f$ is contained in $V_{i j}$, then we have

$$
\begin{aligned}
\sum_{x \in L_{i j}\left(t_{1}, t_{2}\right) / \sim} \mu(x) & =\left(\int_{V\left(t_{1}, t_{2}\right) \boldsymbol{R}} f \cdot \omega\right)^{-1} \int_{G_{+}^{1} / \Gamma} \sum_{x \in L\left(t_{1}, t_{2}\right)} f(\rho(g) x) d^{1} g \\
& =\left(\int_{V\left(t_{1}, t_{2}\right)} f \cdot \omega\right)^{-1} \int_{S L(n: R) / S L(n: Z)} \sum_{x \in L\left(t_{1}, t_{2}\right)} f(\rho(g) x) d^{1} g .
\end{aligned}
$$

Let $H=S L(n), H_{A}$ be the adelization of $H$ and $d H_{A}$ be the canonical Haar measure on $H_{A}$ (i.e., $\int_{H_{A} / H_{Q}} d H_{A}=1$ ). We denote by $\rho_{A}$ the action of $H_{A}$ on the adelization $V_{A}$ of $V_{Q}$. It is easy to see that

$$
\begin{aligned}
\int_{S L(n: R) / S L(n: Z)} & \sum_{x \in L\left(t_{1}, t_{2}\right)} f\left(\rho(g) x d^{1} g\right. \\
= & \int_{H_{A} / H_{\boldsymbol{Q}}} \sum_{x \in V\left(t_{1}, t_{2}\right) \boldsymbol{Q}} F\left(\rho_{A}(g) x\right) d H_{A}(g) \cdot \int_{S L(n . \boldsymbol{R}) / S L(n: Z)} d^{1} g,
\end{aligned}
$$

where $F$ is the Schwarz-Bruhut function on $V_{A}$ defined by the direct product of $f(x)$ and the characteristic functions of $L_{p}$ for all prime numbers $p$. Here $L_{p}$ is the closure of $L$ in $V_{p}=V_{\boldsymbol{Q}} \otimes \boldsymbol{Q}_{P}$. Using the fact that Tamagawa number of the isotropy subgroup of any $Q$-point of $V\left(t_{1}, t_{2}\right)$ is 2 and Hasse principle for the homogeneous space $\left(H, V\left(t_{1}, t_{2}\right)\right)$, we get

$$
\begin{aligned}
\int_{H_{A} / H_{\boldsymbol{Q}}} & \sum_{x \in V\left(t_{1}, t_{2}\right)_{\boldsymbol{Q}}} F\left(\rho_{A}(g) x\right) d H_{A}(g) \\
= & 2 \int_{H_{A} \cdot V\left(t_{1}, t_{2}\right) \boldsymbol{Q}} F \cdot \omega_{A} \leqq 2 \int_{V\left(t_{1}, t_{2}\right)_{A}} F \cdot \omega_{A} \\
= & 2 \int_{V\left(t_{1}, t_{2}\right)_{\boldsymbol{R}}} f \cdot \omega \prod_{p} \int_{L\left(t_{1}, t_{2}\right)_{p}} \omega_{P},
\end{aligned}
$$

where $\omega_{A}$ and $\omega_{p}$ are derived from the differential (gauge) form $\omega$ on $V\left(t_{1}, t_{2}\right)$, and $L\left(t_{1}, t_{2}\right)_{p}$ is the closure of $L\left(t_{1}, t_{2}\right)$ in $V_{p}$. Then we have

$$
\sum_{x \in L_{i j}\left(t_{1}, t_{2}\right) / \sim} \mu(x) \leqq 2 \int_{S L(n: R) / S L(n: Z)} d^{1} g \prod_{p} \int_{L\left(t_{2}, t_{2}\right) p} \omega_{p} .
$$

It is easy to see that there is a finite set $J$ of prime numbers, such that, if $p \notin J$, then $L_{p}=V_{Z_{p}}$, and $H^{(p)}$ and the isotropy subgroup of an 
$\boldsymbol{F}_{p}$-point of $V(1,1)^{(p)}$ are both connected. Here $V_{Z_{p}}=\left\{x=(X, v) \in V_{p}\right.$ : the entries of $X$ and $v$ are containted in $\left.Z_{p}\right\}$. We calculate $\int_{L\left(t_{1}, t_{2}\right)_{p}} \omega_{p}$ in the following cases:

1. $p \in J_{1}=\left\{p \notin J:\left|t_{1}\right|_{p}=1,\left|t_{2}\right|_{p}=1\right\}$,

2. $p \in J_{2}=\left\{p \notin J:\left|t_{1}\right|_{p}<1\right.$ or $\left.\left|t_{2}\right|_{p}<1\right\}$,

3. $p \in J$.

This is easy when $p \in J_{1}$ (see [8]):

$$
\int_{L\left(t_{1}, t_{2}\right)_{p}} \omega_{p}=\int_{V_{Z_{p}}\left(t_{1}, t_{2}\right)} \omega_{p}=p^{-\operatorname{dim} V+2} \sharp\left[V\left(t_{1}, t_{2}\right)^{(p)}\right] .
$$

Here $V_{Z_{p}}\left(t_{1}, t_{2}\right)=\left\{x=(X, v) \in V_{Z_{p}}:|X|=t_{1}, X[v]=t_{2}\right\}$.

When the other cases, we have

$$
\int_{V_{Z_{p}}\left(t_{1}, t_{2}\right)} \omega_{p}=\left|t_{1} t_{2}\right|_{p}^{-n / 2+1} \int_{V_{Z_{p}}\left(t_{1}, t_{2}\right)}\left(\omega_{0}\right)_{p}
$$

and

$$
1=\int_{V_{Z_{p}}} d V_{p} \geqq \int_{\substack{|X| \in t_{1}\left(1+p Z_{p}\right) \\ X[v] \in t_{2}\left(1+p \boldsymbol{Z}_{p}\right)}} d V_{p}=p^{-2} \int_{V_{\boldsymbol{Z}_{p}\left(t_{1}, t_{2}\right)}}\left(\omega_{0}\right)_{p},
$$

where $\left(\omega_{0}\right)_{p}$ is derived from $\omega_{0}$ and $d V_{p}$ is from $d V$. So, when $p \in J_{2}$, by the inequality $p \leqq\left|t_{1} t_{2}\right|_{p}^{-1}$, we have

$$
\int_{L\left(t_{1}, t_{2}\right)_{p}} \omega_{p} \leqq\left|t_{1} t_{2}\right|_{p}^{-n / 2-1}
$$

When $p \in J$, we have

$$
\int_{L\left(t_{1}, t_{2}\right)_{p}} \omega_{p} \leqq c_{p}\left|t_{1} t_{2}\right|_{p}^{-n / 2-1},
$$

where $c_{p}$ is a constant depending on $p$. On the other hand we must show that the product

$$
\prod_{p \in J_{1}} p^{-\operatorname{dim} V+2} \sharp\left[V\left(t_{1}, t_{2}\right)^{(p)}\right]
$$

is dominated by a constant which is independent of $t_{1}$ and $t_{2}$. We know that $V\left(t_{1}, t_{2}\right)$ is a homogeneous space of the special linear group $H=$ $S L(n)$ and the isotropy subgroup $H_{0}$ of a $\boldsymbol{Q}$-rational point of $V\left(t_{1}, t_{2}\right)$ is a special orthogonal group. So, $\left(H, V\left(t_{1}, t_{2}\right)\right)$ is "a special homogeneous space" in the sense of [9]. Further, we have 


$$
p^{-\operatorname{dim} V+2} \sharp\left[V\left(t_{1}, t_{2}\right)^{(p)}\right]=p^{-\operatorname{dim} H} \sharp\left[H^{(p)}\right] / p^{-\operatorname{dim} H_{0}} \sharp\left[H_{0}^{(p)}\right]
$$

where $p \in J_{1}$.

LEMMA 4-1 (Chevally and Steinberg). Let $H$ be a linear algebraic semisimple group (defined over $\boldsymbol{Q}$ ) and $H_{c}$ be a maximal compact subgroup of $H$. We have the following inequalities for primes $p$ (if $H^{(p)}$ is defined):

$$
\prod_{i=1}^{\ell}\left(1-p^{-a_{i}}\right) \leqq p^{-\operatorname{dim} H} \sharp\left[H^{(p)}\right] \leqq \prod_{i=1}^{\ell}\left(1+p^{-a_{i}}\right),
$$

where $a_{i} \geqq 2$ for all $i$ and $\ell$ is the rank of $H_{c}$. Here $a_{i}$ 's are determined by the Betti numbers $b_{\nu}$ of $H_{c}$, i.e.,

$$
\sum_{\nu=0}^{k} b_{\nu} t=\prod_{i=1}^{\ell}\left(1+t^{2 a_{i-1}}\right) \text {. }
$$

Proof. See [7] and [10].

It is a straight consequence of Lemma 4-1 that there exists a constant $C_{0}$ which is independent of $t_{1}$ and $t_{2}$ such that

$$
\prod_{p \in J_{1}} p^{-\operatorname{dim} V+2} \sharp\left[V\left(t_{1}, t_{2}\right)^{(p)}\right]<C_{0} .
$$

By (4-4), (4-4)' and the above inequality we have

$$
\prod_{p} \int_{L\left(t_{1}, t_{2}\right)_{p}} \omega_{p}<C_{0}^{\prime}\left|t_{1} t_{2}\right|^{n / 2+1}
$$

Then we obtain (4-1) by (4-2) and (4-5).

For $(4-1)^{\prime}$, we can apply similar arguments.

Next, we consider integral expressions of "zeta functions" and prove their analytic continuation and functional equation.

We define the following integrals:

$$
\begin{aligned}
& Z\left(f, L, s_{1}, s_{2}\right)=\int_{G^{+} / \Gamma} \chi_{1}(g)^{s_{1}} \chi_{2}(h)^{s_{2}} \sum_{x \in L^{\prime}} f(\rho(g, h) x) d g d h, \\
& \bar{Z}\left(\bar{f}, \bar{L}, s_{1}, s_{2}\right)=\int_{G^{+} / \Gamma} \chi_{1}(g)^{-s_{1}} \chi_{2}(h)^{s_{2}} \sum_{\bar{x} \in \bar{L}^{\prime}} \bar{f}(\bar{\rho}(g, h) \bar{x}) d g d h
\end{aligned}
$$

and

$$
z^{*}\left(f^{*}, L^{*}, s_{1}, s_{2}\right)=\int_{G^{+} / \Gamma} \chi_{1}(g)^{-s_{1}} \chi_{2}(h)^{-s_{2}} \sum_{x^{*} \in\left(L^{*}\right)^{\prime}} f^{*}\left(\rho^{*}(g, h) x^{*}\right) d g d h,
$$

where $f(x) \in \mathscr{S}(V), \bar{f}(\bar{x}) \in \mathscr{S}(\bar{V}), f^{*}\left(x^{*}\right) \in \mathscr{S}\left(V^{*}\right)$. 
LEMMA 4-2. We have the following integral expressions of "zeta functions" :

$$
\begin{aligned}
Z\left(f, L, s_{1}, s_{2}\right)= & \sum_{\substack{0 \leq i \leq n \\
j= \pm 1}} \xi_{i j}\left(s_{1}, s_{2}, L\right) \cdot \Phi_{i j}\left(f, s_{1}-\frac{n}{2}, s_{2}-\frac{n}{2}\right), \\
& \left(\operatorname{Re}\left(s_{1}\right)>A, \operatorname{Re}\left(s_{2}\right)>B\right),
\end{aligned}
$$

$(4-6)^{\prime} \quad \bar{Z}\left(\bar{f}, \bar{L}, s_{1}, s_{2}\right)=\sum_{\substack{0 \leq i \leq n \\ j= \pm 1}} \bar{\xi}_{i j}\left(s_{1}-s_{2}, s_{2}, \bar{L}\right) \cdot \bar{\Phi}_{i j}\left(\bar{f}, s_{1}-s_{2}-1, s_{2}-\frac{n}{2}\right)$,

$$
\left(\operatorname{Re}\left(s_{1}-s_{2}\right)>A, \operatorname{Re}\left(s_{2}\right)>B\right),
$$

$$
\begin{aligned}
(4-6)^{\prime \prime} Z^{*}\left(f^{*}, L^{*}, s_{1}, s_{2}\right)=\sum_{\substack{0 \leq i \leq n \\
j= \pm 1}} \xi_{i j}\left(s_{1}, s_{2}, L^{*}\right) \cdot \Phi_{i j}^{*}\left(f^{*}, s_{1}-\frac{n}{2}, s_{2}-\frac{n}{2}\right), \\
\left(\operatorname{Re}\left(s_{1}\right)>A, \operatorname{Re}\left(s_{2}\right)>B\right) .
\end{aligned}
$$

Proof. We only show the case of $\bar{Z}$. We have

$$
\begin{aligned}
\bar{Z}\left(\bar{f}, \bar{L}, s_{1}, s_{2}\right) & =\int_{G^{+} / G_{+}^{1}} \chi_{1}(\dot{g})^{-s_{1}} \chi_{2}(h)^{s_{2}} \frac{d \chi_{1}(\dot{g})}{\chi_{1}(\dot{g})} \frac{d \chi_{2}(h)}{\chi_{2}(h)} \int_{G_{+}^{1} / \Gamma} \sum_{\bar{x} \in \bar{L}^{\prime}} \bar{f}(\bar{\rho}(\dot{g} g, h) \bar{x}) d^{1} g \\
& =\sum_{\bar{x} \in \bar{L}^{\prime} / \sim} \mu(\bar{x}) \int \chi_{1}(\dot{g})^{-s_{1}} \chi_{2}(h)^{s_{2}} \frac{d \chi_{1}(\dot{g})}{\chi_{1}(\dot{g})} \frac{d \chi_{2}(h)}{\chi_{2}(h)} \int_{p_{\left(G_{+}^{1}\right) \cdot \bar{x}}} \bar{f}_{(\dot{g}, h) \cdot \bar{\omega},}
\end{aligned}
$$

where $\bar{f}_{(\dot{g}, h)}(\bar{x})=\bar{f}(\bar{\rho}(\dot{g}, h) \bar{x})$. It is easy to see that

$$
\begin{aligned}
\int_{\bar{\rho}\left(G_{+}^{1}\right) \cdot \bar{x}} \bar{f}_{(\dot{g}, h)} \bar{\omega} & =\int_{p\left(G_{+}^{1}\right)\langle(\dot{g}, h) \bar{x})} \bar{f} \cdot \bar{\omega} \\
& =\bar{\Psi}_{i j}\left(\bar{f}, \chi_{1}(\dot{g})^{-1}\|Y\|, \chi_{1}(\dot{g})^{-1} \chi_{2}(h) \cdot\left|Y^{c}[v]\right|\right),
\end{aligned}
$$

when $\bar{x}=(Y, v) \in V_{i j},(0 \leqq i \leqq n, j= \pm 1)$. And we have

$$
\begin{gathered}
\bar{Z}\left(\bar{f}, \bar{L}, s_{1}, s_{2}\right)=\sum_{\bar{x} \in \bar{L}^{\prime} / \sim} \mu(\bar{x}) \cdot \bar{\Psi}_{i j}\left(\bar{f}, \chi_{1}(\dot{g})^{-1}\|Y\|, \chi_{1}(\dot{g})^{-1} \chi_{2}(h) \cdot\left|Y^{c}[v]\right|\right) \\
\cdot \int_{G^{+} / G_{+}^{1}} \chi_{1}(\dot{g})^{-s_{1}} \chi_{2}(h)^{s_{2}} \frac{d \chi_{1}(\dot{g})}{\chi_{1}(\dot{g})} \frac{d \chi_{2}(h)}{\chi_{2}(h)} \\
=\sum_{\bar{x} \in \bar{L}^{\prime} / \sim} \mu(\bar{x}) \int_{0}^{\infty} \int_{0}^{\infty} \bar{\Psi}_{i j}\left(\bar{f}, t_{1}, t_{2}\right)\|Y\|^{-s_{1}} \cdot t_{1}^{s_{1}} \\
\cdot\left|Y^{c}[v]\right|^{-s_{2}} \cdot t_{2}^{s_{2}} \cdot\|Y\|^{s_{2}} \cdot t_{1}^{s_{2}} \frac{d t_{1}}{t_{1}} \frac{d t_{2}}{t_{2}} \\
=\sum_{\substack{0 \leqq i \leq n \\
j= \pm 1}} \sum_{\bar{x} \in \bar{L}_{i j /}} \mu(\bar{x})\|Y\|^{-s_{1}+s_{2}}\left|Y^{c}[v]\right|^{-s_{2}} \\
\qquad \int_{0}^{\infty} \int_{0}^{\infty} \bar{\Psi}_{i j}\left(\bar{f}, t_{1}, t_{2}\right) t_{1}^{s_{1}-s_{2}} \cdot t_{2}^{s_{2}} \frac{d t_{1}}{t_{1}} \frac{d t_{2}}{t_{2}} \\
=\sum_{\substack{0 \leq \Sigma \leq n \\
\bar{j}= \pm 1}} \bar{\xi}_{i j}\left(s_{1}-s_{2}, s_{2}, \bar{L}\right) \cdot \bar{\Phi}_{i j}\left(\bar{f}, s_{1}-s_{2}-1, s_{2}-\frac{n}{2}\right) .
\end{gathered}
$$


The above calculations are justified if $\operatorname{Re}\left(s_{1}-s_{2}\right)>A$ and $\operatorname{Re}\left(s_{2}\right)>B$.

Now we prove the functional equation. For $\bar{f}(\bar{x}) \in \mathscr{S}(\bar{V})$, put

$$
f(x)=f(X, v)=\int_{U^{*}} \bar{f}(Y, v) e[\operatorname{Tr} X Y] d Y,
$$

then we have the Poisson summation formula

$$
\sum_{\bar{x} \in \bar{L}} \bar{f}(\bar{x})=v\left(M^{*}\right)^{-1} \sum_{x \in L} f(x),
$$

where $v\left(M^{*}\right)=\int_{U^{*} / M^{*}} d Y$.

Let $\bar{f}_{0}(\bar{x}) \in \mathscr{C}_{0}^{\infty}(\bar{V}-\bar{S})$ and set $\bar{f}(\bar{x})=|d / d Y|(d / d Y)[v] \cdot \bar{f}_{0}(\bar{x})$, then the function $f(x)=f(X, v)=\int_{U^{*}} \bar{f}(Y, v) e[\operatorname{Tr} X Y] d Y$ vanishes on the hypersurface $S$. We apply the formula (4-7) to the function $\bar{f}(\bar{\rho}(g, h) \bar{x})$ of $\bar{x}$, where $(g, h) \in G^{+}$, then we have

$$
\chi_{1}(g)^{-(n+1) / 2} \sum_{\bar{x} \in \bar{L}} \bar{f}(\bar{\rho}(g, h) \bar{x})=v\left(M^{*}\right)^{-1} \sum_{x \in L^{\prime}} f(\rho(g, h) x) .
$$

It is easy to see that the functions

$$
Z_{+}\left(f, L, s_{1}, s_{2}\right)=\int_{\substack{G^{+}(\Gamma) \\ \chi_{1}(g) \geqq 1}} \chi_{1}(g)^{s_{1}} \chi_{2}(h)^{s_{2}} \sum_{x \in L^{\prime}} f(\rho(g, h) x) d g d h
$$

and

$$
\bar{Z}_{+}\left(\bar{f}, \bar{L}, s_{1}, s_{2}\right)=\int_{\substack{G+/ \Gamma \\ \chi_{1}(g) \leqq 1}} \chi_{1}(g)^{-s_{1}} \chi_{2}(h)^{s_{2}} \sum_{\bar{x} \in \bar{L}^{\prime}} \bar{f}(\rho(g, h) \bar{x}) d g d h
$$

are holomorphically continued to the domain $\left\{\left(s_{1}, s_{2}\right) \in C^{2}: \operatorname{Re}\left(s_{2}\right)>B\right\}$. By (4-8), we can calculate

$$
\begin{aligned}
v\left(M^{*}\right)^{-1} Z\left(f, L, s_{1}, s_{2}\right)=v\left(M^{*}\right)^{-1}\left\{\int_{\substack{G^{+} / \Gamma \\
\chi_{1}(g) \geqq 1}}+\int_{\substack{G+/ \Gamma \\
\chi_{1}(g) \geqq 1}}\right\} \\
\cdot \chi_{1}(g)^{s_{1}} \chi_{2}(h)^{s_{2}} \sum_{\substack{x \in L^{\prime}\\
}} f(\rho(g, h) x) d g d h \\
=v\left(M^{*}\right)^{-1} Z_{+}\left(f, L, s_{1}, s_{2}\right)+\int_{\substack{\alpha_{1}(g) \geqq 1 \\
\chi_{1}\left(\chi_{1}\right.}} \chi_{1}(g)^{s_{1}-(n+1) / 2} \chi_{2}(h)^{s_{2}} \\
=v\left(M^{*}\right)^{-1} Z_{+}\left(f, L, s_{1}, s_{2}\right)+\bar{Z}_{+}\left(\bar{f}, \bar{L}, \frac{n+1}{2}-s_{1}, s_{2}\right) .
\end{aligned}
$$


Similarly we have

$$
\begin{aligned}
\bar{Z}\left(\bar{f}, \bar{L}, \frac{n+1}{2}-s_{1}, s_{2}\right)= & v\left(M^{*}\right)^{-1} Z_{+}\left(f, L, s_{1}, s_{2}\right) \\
& +\bar{Z}_{+}\left(\bar{f}, \bar{L}, \frac{n+1}{2}-s_{1}, s_{2}\right) .
\end{aligned}
$$

And, the following equation holds:

$$
v\left(M^{*}\right)^{-1} Z\left(f, L, s_{1}, s_{2}\right)=\bar{Z}\left(\bar{f}, \bar{L}, \frac{n+1}{2}-s_{1}, s_{2}\right),
$$

which are holomorphic in the domain $\left\{\left(s_{1}, s_{2}\right) \in C^{2}: \operatorname{Re}\left(s_{2}\right)>B\right\}$.

Now we suppose that $\operatorname{supp}\left(\bar{f}_{0}\right) \subset V_{i j}$, then (1-1), (4-6), (4-6)' and (4-11) imply

$$
\begin{aligned}
& \text { (4-12) } \bar{\xi}_{i j}\left(\frac{n+1}{2}-s_{1}-s_{2}, s_{2}, \bar{L}\right) \cdot \bar{\Phi}_{i j}\left(\bar{f}, \frac{n-1}{2}-s_{1}-s_{2}, s_{2}-\frac{n}{2}\right) \\
& =v\left(M^{*}\right)^{-1} \sum_{\substack{0 \leqq i \leq n \\
\ell= \pm 1}} \xi_{k \ell}\left(s_{1}, s_{2}, L\right) \cdot \Phi_{k \ell}\left(f, s_{1}-\frac{n}{2}, s_{2}-\frac{n}{2}\right) \\
& =v\left(M^{*}\right)^{-1}(2 \pi)^{-n s_{1}-s_{2}} \cdot \pi^{n(n-1) / 4} \cdot e\left[\frac{n s_{1}+s_{2}}{2}\right] \Gamma\left(s_{1}\right) \Gamma\left(s_{1}-\frac{1}{2}\right) \\
& \cdots \Gamma\left(s_{1}-\frac{n-2}{2}\right) \Gamma\left(s_{1}+s_{2}-\frac{n-1}{2}\right)\left[\sum_{\substack{0 \leq k \leq n \\
\ell= \pm 1}} A_{k \ell, i j}\left(s_{1}, s_{2}\right)\right. \\
& \left.\cdot \xi_{k \ell}\left(s_{1}, s_{2}, L\right)\right] \Phi_{i f}\left(\bar{f}, \frac{n-1}{2}-s_{1}-s_{2}, s_{2}-\frac{n}{2}\right) \text {. }
\end{aligned}
$$

On the other hand, by (1-3), we have

$$
\begin{aligned}
\bar{\Phi}_{i j}\left(\bar{f}, \frac{n-1}{2}-s_{1}-s_{2}, s_{2}-\frac{n}{2}\right) \\
=j\left(s_{1}+s_{2}-\frac{n-3}{2}\right)\left(s_{1}+s_{2}-\frac{n-1}{2}\right) s_{1}\left(s_{1}-\frac{1}{2}\right) \\
\quad \ldots\left(s_{1}-\frac{n-2}{2}\right) \bar{\Phi}_{i j}\left(\bar{f}_{0}, \frac{n-5}{2}-s_{1}-s_{2}, s_{2}-\frac{n}{2}+1\right) .
\end{aligned}
$$

For any $\left(s_{1}, s_{2}\right) \in C^{2}$, we can find $\bar{f}_{0}(\bar{x}) \in \mathscr{C}_{0}^{\infty}\left(\bar{V}_{i j}\right)$ such that $\bar{\Phi}_{i j}\left(\bar{f}_{0}, s_{1}, s_{2}\right) \neq 0$. So, the functions

$$
\begin{gathered}
\left(s_{1}+s_{2}-\frac{n-3}{2}\right)\left(s_{1}+s_{2}-\frac{n-1}{2}\right) s_{1}\left(s_{1}-\frac{1}{2}\right) \cdots\left(s_{1}-\frac{n-2}{2}\right) \\
\cdot \bar{\xi}_{i j}\left(\frac{n+1}{2}-s_{1}-s_{2}, s_{2}, \bar{L}\right), \quad(0 \leqq i \leqq n, j= \pm 1),
\end{gathered}
$$


are holomorphic in the domain $\left\{\left(s_{1}, s_{2}\right) \in C_{2}: \operatorname{Re}\left(s_{2}\right)>B\right\}$, and (2-4) holds in this domain.

Let $f_{0}(x) \in \mathscr{C}_{0}^{\infty}\left(V_{i j}\right)$ and set $f(x)=|d / d x|(d / d x)^{c}[v] \cdot f_{0}(x)$, then the function $\bar{f}(\bar{x})=\bar{f}(Y, v)=\int_{U} f(X, v) e[\operatorname{Tr} X Y] d X$ vanishes on the hypersurface $\bar{S}$. For this $f(x)$ and $\bar{f}(\bar{x})$, (4-11) holds, too. So, by (1-4), the functions

$$
\begin{aligned}
& \left(s_{1}-1\right)\left(s_{1}-\frac{1}{2}-1\right) \cdots\left(s_{1}-\frac{n-2}{2}-1\right)\left(s_{1}-2\right)\left(s_{1}-\frac{1}{2}-2\right) \\
& \cdots\left(s_{1}-\frac{n-2}{2}-2\right)\left(s_{1}+s_{2}-\frac{n-3}{2}-2\right) \xi_{i j}\left(s_{1}, s_{2}, L\right) \\
& \quad(0 \leqq i \leqq n, j= \pm 1)
\end{aligned}
$$

are holomorphic in the domain $\left\{\left(s_{1}, s_{2}\right) \in C^{2}: \operatorname{Re}\left(s_{2}\right)>B\right\}$.

Next for $f^{*}\left(x^{*}\right) \in \mathscr{S}\left(V^{*}\right)$ and $\bar{f}(\bar{x})=\int_{W^{*}} f^{*}(Y, u) e\left[{ }^{t} u v\right] d u$, we have the Poisson summation formula

$$
\sum_{x * \in L^{*}} f^{*}\left(x^{*}\right)=v(N)^{-1} \sum_{\bar{x} \in \bar{L}} \bar{f}(\bar{x})
$$

We take $f_{0}^{*}\left(x^{*}\right) \in \mathscr{C}_{0}^{\infty}\left(V_{i j}^{*}\right)$ and put $f^{*}\left(x^{*}\right)=Y^{c}[d / d u] \cdot f_{0}^{*}\left(x^{*}\right)$. The following is easy:

$$
\chi_{1}(g)^{1 / 2} \chi_{2}(h)^{-n / 2} \sum_{x^{*} \in\left(L^{*}\right)^{\prime}} f^{*}\left(\rho^{*}(g, h) x^{*}\right)=v\left(N^{*}\right)^{-1} \sum_{\bar{x} \in \bar{L}} \bar{f}(\bar{\rho}(g, h) \bar{x}) .
$$

By calculation similar to (4-9), we have, for the above $f^{*}$ and $\bar{f}$,

$$
v\left(N^{*}\right)^{-1} \bar{Z}\left(\bar{f}, \bar{L}, s_{1}+\frac{1}{2}, \frac{n}{2}-s_{2}\right)=Z^{*}\left(f^{*}, L^{*}, s_{1}, s_{2}\right),
$$

which is holomorphic in the domain $\left\{\left(s_{1}, s_{2}\right) \in C^{2}: \operatorname{Re}\left(s_{1}+s_{2}-(n-1 / 2)\right)\right.$ $\left.>A, \operatorname{Re}\left(s_{1}\right)>A\right\}$.

By (1-2), (4-6)', (4-6)" and (4-14), we obtain

$$
\begin{aligned}
\xi_{i j}\left(s_{1}, s_{2}, L^{*}\right) \cdot \Phi_{i j}^{*}\left(f^{*}, s_{1}-\frac{n}{2}, s_{2}-\frac{n}{2}\right) \\
=v\left(N^{*}\right)^{-1} \pi^{2 s_{2}-n / 2-1} \Gamma\left(\frac{n}{2}-s_{2}\right) \Gamma\left(1-s_{2}\right) \\
\cdot \sum_{\ell= \pm 1} B_{\ell j}^{i}\left(s_{2}\right) \xi_{i \ell}\left(s_{1}+s_{2}-\frac{n-1}{2}, \frac{n}{2}-s_{2}, \bar{L}\right) \\
\cdot \Phi_{i j}^{*}\left(f^{*}, s_{1}-\frac{n}{2}, s_{2}-\frac{n}{2}\right), \quad(0 \leqq i \leqq n, j= \pm 1) .
\end{aligned}
$$


By (1-5) and similar arguments to the former part, we can see that the functions $\left(s_{2}-(n / 2)\right)\left(s_{2}-1\right) \xi_{i j}\left(s_{1}, s_{2}, L^{*}\right),(0 \leqq i \leqq n, j= \pm 1)$ are holomorphic in the domain $\left\{\left(s_{1}, s_{2}\right) \in C^{2}: \operatorname{Re}\left(s_{1}+s_{2}-(n-1 / 2)\right)>A, \operatorname{Re}(s)>\right\}$, and (2-5) holds there.

Similarly, the functions $\left(s_{2}-(n / 2)\right)\left(s_{2}-1\right) \bar{\xi}_{i j}\left(s_{1}-s_{2}, s_{2}\right),(0 \leqq i \leqq n$, $j= \pm 1$ ) are holomorphic in the domain $\left\{\left(s_{1}, s_{2}\right) \in C^{2}: \operatorname{Re}\left(s_{1}-s_{2}\right)>A\right.$, $\left.\operatorname{Re}\left(s_{1}\right)-1 / 2>A\right\}$.

Combining the above results, the functions

$$
\begin{gathered}
\xi_{i j}\left(s_{1}, s_{2}, L\right) \cdot\left(s_{1}+s_{2}-\frac{n-3}{2}\right)\left(s_{1}-1\right)\left(s_{1}-1-\frac{1}{2}\right) \\
\ldots\left(s_{1}-1-\frac{n-2}{2}\right)\left(s_{1}-2\right)\left(s_{1}-2-\frac{1}{2}\right) \\
\ldots\left(s_{1}-2-\frac{n-2}{2}\right)\left(s_{2}-1\right)\left(s_{2}-\frac{n}{2}\right)
\end{gathered}
$$

and

$$
\begin{aligned}
& \bar{\xi}_{i j}\left(s_{1}, s_{2}, \bar{L}\right) \cdot\left(s_{1}-1\right)\left(s_{1}-2\right)\left(s_{1}+s_{2}-1-\frac{1}{2}\right)\left(s_{1}+s_{2}-1-\frac{2}{2}\right) \\
& \ldots\left(s_{1}+s_{2}-1-\frac{n-1}{2}\right)\left(s_{2}-1\right)\left(s_{2}-\frac{n}{2}\right), \\
& \quad(0 \leqq i \leqq n, j= \pm 1)
\end{aligned}
$$

are holomorphically continued to the domains $\left\{\left(s_{1}, s_{2}\right) \in C^{2}: \operatorname{Re}\left(s_{1}+s_{2}\right.\right.$ $\left.-(n-1 / 2))>A, \operatorname{Re}\left(s_{1}\right)>A\right\} \cup\left\{\left(s_{1}, s_{2}\right) \in C^{2}: \operatorname{Re}\left(s_{2}\right)>B\right\}$. So, they are holomorphic on the whole plane $C^{2}$, and (2-4), (2-5) hold on the whole plane $\boldsymbol{C}^{2}$.

\section{Some residues}

In this section, we shall study some residues of the zeta functions $\xi_{i j}\left(s_{1}, s_{2}, L\right)$ and $\bar{\xi}_{i j}\left(s_{1}, s_{2}, \bar{L}\right),(0 \leqq i \leqq n, j= \pm 1)$.

The triplet $(G, \rho, V)$ induces the action of $G L(n ; R) \subset G$ on the subset $R-P$ of $V$, whose complexification is prehomogeneous. We decompose $(G L(n ; \boldsymbol{R}), R-P)$ into two spaces $(G L(n ; R), R-P-U \oplus\{0\})$ and $(G L(n ; \boldsymbol{R})$, $U \oplus\{0\}-P)$, whose complexifications are homogeneous. The zeta functions associated with the vector space of quadratic forms, $\xi_{i}(s),(0 \leqq i \leqq n)$, is obtained from the latter space, (see [2]). We define zeta functions $\xi_{i}^{\prime}(s),(1 \leqq i \leqq n-1)$, attached to the former space $(G L(n ; R), R-P-U$ 
$\oplus\{0\})$. For the sake of brevity, we write $R$ instead of $R-P-U \oplus\{0\}$. Similarly, we write $\bar{R}$ and $R^{*}$ instead of $\bar{R}-\bar{P}-U^{*} \oplus\{0\}$ and $R^{*}-P^{*}$ $-U^{*} \oplus\{0\}$, respectively.

Let $R_{i}^{*}=\left\{x^{*}=(Y, u) \in R^{*}: \operatorname{sgn} Y=(i, n-i)\right\}$ and $\bar{R}_{i}=\{\bar{x}=(Y, v)$ $\in \bar{R}: \operatorname{sgn} Y=(i, n-i)\},(1 \leqq i \leqq n-1)$, then we have $R^{*}=\cup R_{i}^{*}$ and $\bar{R}=\cup \bar{R}_{i}$, which are the orbital decompositions of $R^{*}$ and $\bar{R}$ by the group $G L(n ; \boldsymbol{R})$, respectively.

We define the measures $d R^{*}$ and $d \bar{R}$ on $R^{*}$ and $\bar{R}$ by

$$
d R^{*}=d Y \cdot\left|\frac{d Y[u]}{d u_{1}}\right|^{-1} d u_{2} \cdots d u_{n}=d(\|Y\|) \cdot \omega_{0}^{*}, \quad\left(u={ }^{t}\left(u_{1}, \cdots, u_{n}\right)\right)
$$

and

$$
d \bar{R}=d Y \cdot\left|\frac{d Y^{c}[v]}{d v_{1}}\right|^{-1} d v_{2} \cdots d v_{n}=d(\|Y\|) \cdot \bar{\omega}_{0}, \quad\left(v={ }^{t}\left(v_{1}, \cdots, v_{n}\right)\right),
$$

respectively. (It is easy to see that $\omega_{0}^{*}$ and $\bar{\omega}_{0}$ can be defined on a neighborhood of any generic point of the hypersurface $S$.) Let $R_{i}^{*}(t)=\{(Y, u)$ $\left.\in R_{\imath}^{*}:|Y|=t\right\}$ and $\bar{R}_{i}(t)=\left\{(Y, v) \in \bar{R}_{i}:|Y|=t\right\}$, for a real number $t \neq 0$. We define the differential forms $\omega_{R}^{*}$ and $\bar{\omega}_{R}$ on $R^{*}$ and $\bar{R}$ by $\omega_{R}^{*}=\|Y\|^{-(n-2) / 2} \omega_{0}^{*}$ and $\bar{\omega}_{R}=\|Y\|^{-(n-2) / 2} \bar{\omega}_{0}$, respectively. They may be considered the measures on $R_{i}^{*}(t)$ and $\bar{R}_{i}(t)$, respectively.

For any $x^{*} \in R_{i}^{*}(t)$ and $\bar{x} \in \bar{R}_{i}(t)$, there exist Haar measures $d \nu_{x^{*}}$ and $d \nu_{\bar{x}}$ on the isotropy subgroups $G^{+}\left(x^{*}\right)$ and $G^{+}(\bar{x})$ of $G_{+}^{1} \subset G L(n ; R)$ such that

$$
\int_{G_{+}^{1}} f_{1}(g) d^{1} g=\int_{R_{i}^{*}(t)} \omega_{R}^{*}\left(Y\left[\dot{g}^{-1}\right], \dot{g} u\right) \cdot \int_{G^{+}\left(x^{*}\right)} f_{1}(\dot{g} g) d \nu_{x^{*}}(g)
$$

and

$$
\begin{aligned}
& \int_{G_{+}^{1}} f_{1}(g) d^{1} g=\int_{\bar{R}_{i}(t)} \bar{\omega}_{R}\left(Y\left[\dot{g}^{-1}\right],{ }^{t} \dot{g}^{-1} v\right) \cdot \int_{G^{+}(\bar{x})} f_{1}(\dot{g} g) d \nu_{\bar{x}}(g), \\
&\left(f_{1} \in L_{1}\left(G_{+}^{1}\right)\right),
\end{aligned}
$$

respectively.

The zeta functions $\xi_{i}^{\prime}\left(s, L^{*}\right)$ and $\bar{\xi}_{i}^{\prime}(s, \bar{L}),(1 \leqq i \leqq n-1)$, are defined by

$$
\xi_{i}^{\prime}\left(s, L^{*}\right)=\sum_{x^{*} \in R_{i}^{*} \cap L^{* / \sim}} \mu\left(x^{*}\right)\|Y\|^{-s}
$$

and 


$$
\bar{\xi}_{i}^{\prime}(s, \bar{L})=\sum_{\bar{x} \in \bar{R}_{i} \cap \bar{L} / \sim} \mu(\bar{x})\|Y\|^{-s}, \quad(1 \leqq i \leqq n-1),
$$

where

$$
\mu\left(x^{*}\right)=\int_{G+\left(x^{*}\right) / \Gamma^{*}} d \nu_{x^{*}}\left(\Gamma_{x^{*}}=G^{+}\left(x^{*}\right) \cap I^{\urcorner}\right)
$$

and

$$
\mu(\bar{x})=\int_{G^{+}(\bar{x}) / \Gamma \bar{x}} d \nu_{\bar{x}}\left(\Gamma_{\bar{x}}=G^{+}(\bar{x}) \cap \Gamma\right)
$$

respectively.

We show that there exists a positive number $K$ such that, if $n \geqq 5$ and $\operatorname{Re}(s)>K$, then $\xi_{i}^{\prime}\left(s, L^{*}\right)$ and $\bar{\xi}_{i}^{\prime}(s, \bar{L}),(1 \leqq i \leqq n-1)$, are absolutely convergent, i.e., there exist positive numbers $K^{\prime}$ and $C$ such that

$$
\begin{gathered}
\sum_{x^{*} \in R_{i}^{*}(t) \cap L^{*} / \sim} \mu\left(x^{*}\right)<C|t|^{K^{\prime}} \\
\sum_{\bar{x} \in \bar{R}_{i}(t) \cap \bar{L} / \sim} \mu(\bar{x})<C|t|^{K^{\prime}}
\end{gathered}
$$

where $t$ is a rational number.

For any rational number $t \neq 0$, set $R^{*}(t)=\left\{x^{*} \in\left(R^{*}\right)^{c}:|Y|=t\right\}$ which are affine varieties defined over $\boldsymbol{Q}$. Suppose $n \geqq 5$ and consider the special homogeneous space $\left(H, R^{*}(t)\right)$. By similar arguments to the former part of section 4 , we have

$$
\sum_{x^{*} \in R_{i}^{*}(t) \cap L^{*} / \sim} \mu\left(x^{*}\right) \leqq 2 \int_{G_{+}^{1} / \Gamma} d^{1} g \cdot \prod_{p} \int_{\left(R^{*}(t) \cap L^{*}\right)_{p}}\left(\omega_{R}^{*}\right)_{p},
$$

where $\left(R^{*}(t) \cap L^{*}\right)_{p}$ is the closure of $R^{*}(t) \cap L^{*}$ in $V_{p}^{*}$ and $\left(\omega_{R}^{*}\right)_{p}$ is derived from $\omega_{R}^{*}$. There is a finite set $J$ of prime numbers such that, if $p \notin J$, then $L_{p}^{*}=V_{Z_{p}}^{*}$, and $H^{(p)}$ and the isotropy subgroup of an $F_{p}$-point of $R^{*}(t)^{(p)}$ are both connected. Further, by Lang-Weil theorem, we can choose $J$ such that $p \geqq p^{-\operatorname{dim} V^{+1}}\left[R^{*(p)}\right]$ if $p \notin J$ (see [12]). When $|t|_{p}=1$ for $p \notin J$, we have

$$
\int_{\left(R^{*}(t) \cap L^{*}\right)_{p}}\left(\omega_{R}^{*}\right)_{p}=p^{-\operatorname{dim} V+2} \sharp\left[R^{*}(t)^{(p)}\right] .
$$

When $|t|_{p}<1$ for $p \notin J$, we have

$$
\int_{\left(R^{*}(t) \cap L^{*}\right) p}\left(\omega_{R}^{*}\right)_{p} \leqq|t|_{p}^{-n / 2},
$$

because 


$$
p^{-\operatorname{dim} V+1} \sharp\left[R^{*(p)}\right]=\int_{R_{Z_{p}^{*}}^{*}}\left(d R^{*}\right)_{p} \geqq \int_{\substack{R_{Z_{p}}^{*} \\|Y| \in t\left(1+p Z_{p}\right)}}\left(d R^{*}\right)_{p}=p^{-1} \int_{R^{*}(t) Z_{p}}\left(\omega_{0}^{*}\right)_{p},
$$

where $\left(d R^{*}\right)_{p}$ is derived from $d R^{*}$.

And other parts follow after similar arguments to section 4 . We can identify $U^{*} \oplus\{0\}$ with $U^{*}$. Let $U_{i}^{*}=\left\{Y \in U^{*}:|Y| \neq 0, \operatorname{sgn} Y=\right.$ $(i, n-i)\}$ be considered the submanifold of $V^{*}$ and $\bar{V},(o \leqq i \leqq n)$.

Let $f^{*} \in \mathscr{C}_{0}^{\infty}\left(V_{i j}^{*}\right)$ and $\bar{f}(\bar{x})=\int_{W^{*}} f^{*}(Y, u) e\left[{ }^{t} v u\right] d u$. Then, $\operatorname{supp}(\bar{f})$ is contained in $\left(\bigcup_{j= \pm 1} \bar{V}_{i j}\right) \cup \bar{R}_{i} \cup U_{i}^{*} \subset \bar{V}-\bar{P}$. At least formally, we show the following by routine arguments ([1]) :

$$
\begin{aligned}
& \text { (5-4) } \xi_{i j}\left(s_{1}, s_{2}, L^{*}\right) \cdot \Phi_{i j}^{*}\left(f^{*}, s_{1}-\frac{n}{2}, s_{2}-\frac{n}{2}\right) \\
& =Z^{*+}\left(f^{*}, L^{*}, s_{1}, s_{2}\right)+v\left(N^{*}\right)^{-1} \bar{Z}^{+}\left(\bar{f}, \bar{L}, s_{1}+\frac{1}{2}, \frac{n}{2}-s_{2}\right) \\
& +v\left(N^{*}\right)^{-1} \int_{\substack{G+\Gamma>\\
\chi_{2}(h) \leqq 1}} \chi_{1}(g)^{-s_{1}-(1 / 2)} \chi_{2}(h)^{(n / 2)-s_{2}} \sum_{\bar{x} \in \bar{R}_{i \cap} \cap \bar{L}} \bar{f}(\bar{\rho}(g, h) \bar{x}) d g d h \\
& +v\left(N^{*}\right)^{-1} \int_{\substack{G+/ \Gamma \\
\chi_{2}(h) \leqq 1}} \chi_{1}(g)^{-s_{1}-(1 / 2)} \chi_{2}(h)^{(n / 2)-s_{2}} \sum_{\bar{x} \in U_{i}^{*} \cap \bar{L}} \bar{f}(\bar{\rho}(g, h) \bar{x}) d g d h,
\end{aligned}
$$

where

$$
Z^{*+}\left(f^{*}, L^{*}, s_{1}, s_{2}\right)=\int_{\substack{G+/ \Gamma \\ \chi_{2}(h) \leqq 1}} \chi_{1}(g)^{-s_{1}} \chi_{2}(h)^{-s_{2}} \sum_{x^{*} \in\left(L^{*}\right)^{\prime}} f^{*}\left(\rho^{*}(g, h) x^{*}\right) d g d h
$$

and

$$
\bar{Z}^{+}\left(\bar{f}, \bar{L}, s_{1}, s_{2}\right)=\int_{\substack{G^{+} / r \\ \chi_{2}(h) \geqq 1}} \chi_{1}(g)^{-s_{1}} \chi_{2}(h)^{s_{2}} \sum_{\bar{x} \in \bar{L}^{\prime}} \bar{f}(\bar{\rho}(g, h) \bar{x}) d g d h .
$$

Using the results of [1], $\S 2,4^{0}$, we calculate the following integral, which is absolutely convergent if $\operatorname{Re}\left(s_{1}\right)>K$ :

$$
\begin{aligned}
& \text { (5-5) } \int_{\substack{G+/ \Gamma \\
\chi_{2}(h) \leqq 1}} \chi_{1}(g)^{-s_{1}-(1 / 2)} \chi_{2}(h)^{(n / 2)-s_{2}} \sum_{\bar{x} \in \bar{R}_{i} \cap \bar{L}} \bar{f}(\bar{\rho}(g, h) \bar{x}) d g d h \\
& =\int_{\substack{G+/ G_{+}^{1} \\
\chi_{2}(h) \leqq 1}} \chi_{1}(\dot{g})^{-s_{1}-(1 / 2)} \chi_{2}(h)^{(n / 2)-s_{2}} \frac{d \chi_{1}(\dot{g})}{\chi_{1}(\dot{g})} \frac{d \chi_{2}(h)}{\chi_{2}(h)} \cdot \int_{\bar{x} \in \bar{R}_{i} \cap \bar{L}} \bar{f}\left(\bar{\rho}(\dot{g} g, h) \bar{x}^{1}\right) d^{1} g \\
& =\left(s_{2}-1\right)^{-1} \sum_{\bar{x} \in \bar{R}_{i} \cap \bar{L} / \sim} \mu(\bar{x})\|Y\|^{-s_{1}-(1 / 2)} \cdot \int_{\bar{R}_{i}} \bar{f}(\bar{x})\|Y\|^{s_{1}-(n-1 / 2)} d \bar{R}
\end{aligned}
$$




$$
\begin{aligned}
&=\left(s_{2}-1\right)^{-1} \xi_{i}^{\prime}\left(s_{1}+\frac{1}{2}, \bar{L}\right) \cdot \int_{U^{*}} \| Y \|^{s_{1}-(n-1 / 2)} d Y \\
& \cdot \int_{Y^{c}[v]=0} \bar{f}(\bar{x})\left|\frac{d Y^{c}[v]}{d v_{1}}\right| d v_{2} \cdots d v_{1} \\
&=\left(s_{2}-1\right)^{-1} \xi_{i}^{\prime}\left(s_{1}+\frac{1}{2}, \bar{L}\right) \cdot \pi^{1-(n / 2)} \Gamma\left(\frac{n}{2}-1\right) \\
& \cdot \int_{V_{i j}^{*} j} f^{*}\left(x^{*}\right)\|Y\|^{s_{1}-(n / 2)}|Y[u]|^{-(n-2 / 2)} d Y d u \\
& \times \begin{cases}\sin \pi(n-i) / 2 & \text { when } j=+1, \\
\sin \pi i / 2 & \text { when } j=-1 .\end{cases}
\end{aligned}
$$

Similarly, using the results of [2], chap 2, we have

$$
\begin{aligned}
\int_{\substack{G+/ \Gamma \\
\chi_{2}(h) \leqq 1}} \chi_{1}(g)^{-s_{1}-(1 / 2)} \chi_{2}(h)^{(n / 2)-s_{2}} \sum_{\bar{x} \in U_{i}^{*} \cap \bar{L}} \bar{f}(\bar{\rho}(g, h) \bar{x}) d g d h \\
=\left(s_{2}-\frac{n}{2}\right)^{-1} \cdot \xi_{i}\left(s_{1}+\frac{1}{2}, M^{*}\right) \cdot \int_{U_{i}^{*}} \bar{f}(Y, 0)\|Y\|^{s_{1}-(n / 2)} d Y \\
=\left(s_{2}-\frac{n}{2}\right)^{-1} \cdot \xi_{i}\left(s_{1}+\frac{1}{2}, M^{*}\right) \cdot \int_{V_{i j}^{*}} f^{*}\left(x^{*}\right)\|Y\|^{s_{1}-(n / 2)} d x^{*} .
\end{aligned}
$$

We can see that (5-4) is justified in the domain $\left\{\left(s_{1}, s_{2}\right) \in C^{2}: \operatorname{Re}\left(s_{1}\right)>A\right.$, $\left.\operatorname{Re}\left(s_{1}+(1 / 2)-(n / 2)+s_{2}\right)>A, \operatorname{Re}\left(s_{1}+(1 / 2)\right)>\operatorname{Max}\{K,(n+1) / 2\}\right\}$. So, (5-5) and (5-6) imply

$$
\begin{aligned}
&\left.\left(s_{2}-\frac{n}{2}\right) \xi_{i j}\left(s_{1}, s_{2}, L^{*}\right)\right|_{s_{2}=n / 2}=v\left(N^{*}\right)^{-1} \xi_{i}^{*}\left(s_{1}+\frac{1}{2}, M^{*}\right), \\
&\left(0 \leqq i \leqq n, \operatorname{Re}\left(s_{1}\right)>\operatorname{Max}\left\{A, K-\frac{1}{2}, \frac{n}{2}\right\}\right)
\end{aligned}
$$

and

$$
\begin{array}{r}
\left.\left(s_{2}-1\right) \xi_{i j}\left(s_{1}, s_{2}, L^{*}\right)\right|_{s_{2}=1}=v\left(N^{*}\right)^{-1} \xi_{i}^{\prime}\left(s_{1}+\frac{1}{2}, \bar{L}\right) \pi^{1-(n / 2)} \Gamma\left(\frac{n}{2}-1\right) \\
\cdot \begin{cases}\sin \pi(n-i) / 2 & \text { when } j=+1, \\
\sin \pi i / 2 & \text { when } j=-1,\end{cases} \\
\left(1 \leqq i \leqq n-1, \operatorname{Re}\left(s_{1}\right)>\left\{\operatorname{Max} A+\frac{n-3}{2}, M-\frac{1}{2}, \frac{n}{2}\right\}\right)
\end{array}
$$

Similarly, for sufficiently large $\operatorname{Re}\left(s_{1}\right)$, we have

$$
\left.\left(s_{2}-\frac{n}{2}\right) \bar{\xi}_{i j}\left(s_{1}, s_{2}, \bar{L}\right)\right|_{s_{2}=n / 2}=v\left(N^{*}\right)^{-1} \xi_{i}\left(s_{1}+\frac{n+1}{2}, M\right),
$$


$\left.(5-7)^{\prime \prime} \quad\left(s_{2}-\frac{n}{2}\right) \xi_{i j}\left(s_{1}, s_{2}, L\right)\right|_{s_{2}=n / 2}=v(N)^{-1} \xi_{i}\left(s_{1}+\frac{1}{2}, M\right)$,

$$
(0 \leqq i \leqq n, j= \pm 1)
$$

and

$$
\begin{array}{r}
\left.(5-8)^{\prime}\left(s_{2}-1\right) \bar{\xi}_{i j}\left(s_{1}, s_{2}, \bar{L}\right)\right|_{s_{2}=1}=v\left(N^{*}\right)^{-1} \bar{\xi}_{i}^{\prime}\left(s_{1}-\frac{n-1}{2}, \bar{L}\right) \pi^{1-(n / 2)} \Gamma\left(\frac{n}{2}-1\right) \\
\cdot \begin{cases}\sin \pi(n-i) / 2 & \text { when } j=(-1)^{n-i}, \\
\sin \pi i / 2 & \text { when } j=(-1)^{n-i+1},\end{cases} \\
\left.(5-8)^{\prime \prime}\left(s_{2}-1\right) \xi_{i j}\left(s_{1}, s_{2}, L\right)\right|_{s_{2}=1}=v(N)^{-1} \xi_{i}^{\prime}\left(s_{1}-\frac{1}{2}, M \oplus N^{*}\right) \pi^{1-(n / 2)} \Gamma\left(\frac{n}{2}-1\right) \\
\cdot \begin{cases}\sin \pi(n-i) / 2 & \text { when } j=+1, \\
\sin \pi i / 2 & \text { when } j=-1, \\
(1 \leqq i \leqq n-1) .\end{cases}
\end{array}
$$

Now it is clear that $\xi_{i}^{\prime}\left(s, L^{*}\right)$ and $\bar{\xi}_{i}^{\prime}(s, \bar{L})(1 \leq i \leq n-1)$ are continued to meromorphic functions of $s$ on the whole plane $C$.

THEOREM 3. For $n \geqq 5$, the zeta functions $\xi_{i}^{\prime}\left(s, L^{*}\right)$ and $\bar{\xi}_{i}^{\prime}(s, \bar{L})$ $(1 \leqq i \leqq n-1$, and, if $n$ is even, $i$ is odd.) are absolutely convergent if $\operatorname{Re}(s)>K$, and continued to meromorphic functions of $s$ on the whole plane $C$ satisfying the following functional equation:

$$
\begin{gathered}
\xi_{i}^{\prime}\left(\frac{n}{2}-1-s, L^{*}\right)=v\left(M^{*}\right)^{-1}(2 \pi)^{-n s-1} e\left[\frac{n s+1}{4}\right] \Gamma(s) \Gamma\left(s-\frac{1}{2}\right) \\
\cdots \Gamma\left(s-\frac{n-2}{2}\right) \Gamma\left(s-\frac{n-3}{2}\right) \cdot \pi^{n(n-1) / 4} \sum_{1 \leq k \leq n-1} D_{i k}(s) \\
\cdot \xi_{k}^{\prime}\left(s+\frac{1}{2}, M \oplus N^{*}\right)
\end{gathered}
$$

where

$$
\begin{aligned}
D_{i k}(s) & =A_{k,-1, i,-1}(s, 1) \quad \text { when } n, i \text { odd }, \\
D_{i k}(s) & =A_{k,+1, i,-1}(s, 1) \quad \text { when } n \text { odd, } i \text { even }, \\
D_{i k}(s) & =A_{k,+1, i,-1}(s, 1)+A_{k,-1, i,-1}(s, 1) \\
& =A_{k,+1, i,+1}(s, 1)+A_{k,-1, i,+1}(s, 1)=v_{k i}^{(n)}\left(s+\frac{1}{2}\right)
\end{aligned}
$$

when $n \equiv 2(4), i$ odd

$$
D_{i k}(s)=A_{k,+1, i,-1}(s, 1)-A_{k,-1, i,-1}(s, 1)
$$




$$
=-A_{k,+1, i,+1}(s, 1)+A_{k,-1, i,+1}(s, 1)=v_{k, i}^{(n)}\left(s+\frac{1}{2}\right)
$$

when $n \equiv 0(4), i$ odd .

Proof. Because, by Theorem 2 and the above results, we obtain the following equations:

$$
\begin{aligned}
& \left\{\begin{array}{l}
\sin \pi(n-i) / 2 \\
\sin \pi i / 2
\end{array}\right\} \cdot \bar{\xi}_{i}^{\prime}\left(\frac{n}{2}-1-s_{1}, L^{*}\right) \\
& =v\left(M^{*}\right)^{-1}(2 \pi)^{-n s_{1}-1} e\left[\frac{n s_{1}+1}{4}\right] \pi^{n(n-1) / 4} \Gamma\left(s_{1}\right) \Gamma\left(s_{1}-\frac{1}{2}\right) \\
& \cdots \Gamma\left(s_{1}-\frac{n-2}{2}\right) \Gamma\left(s_{1}-\frac{n-3}{2}\right) \\
& \sum_{1 \leqq k \leqq n-1}\left\{\begin{array}{l}
A_{k,+1, i(-1)^{n-1}}\left(s_{1}, 1\right) \sin \pi \frac{n-i}{2}+A_{k,-1, i(-1)^{n-i}}\left(s_{1}, 1\right) \sin \pi \frac{i}{2} \\
A_{k,+1, i(-1)^{n-i+1}}\left(s_{1}, 1\right) \sin \pi \frac{n-i}{2}+A_{k,-1, i(-1)^{n-i+1}}\left(s_{1}, 1\right) \sin n \frac{i}{2}
\end{array}\right\} \\
& \cdot \xi_{k}^{\prime}\left(s_{1}+\frac{1}{2}, M \oplus N^{*}\right), \quad(1 \leqq i \leqq n-1) \text {. }
\end{aligned}
$$

\section{REFERENCES}

[1] M. Sato and T. Shintani, On zeta functions associated with prehomogeneous vector space, Ann. of Math. 100 (1974), 131-170.

[2] T. Shintani, On zeta functions associated with the vector space of quadratic forms, J. Fac. Science Univ. of Tokyo Sec. IA, Vol. 22, 25-65.

[ 3 ] - On Dirichlet series whose coefficients are class-numbers of integral binary cubic forms, J. Math. Soc. Japan 24 (1972), 132-188.

[4] M. Sato, Theory of prehomogeneous vector spaces (notes by T. Shintani in Japanese), Sugaku no Ayumi 15 (1970), 85-157.

[5] I. M. Gel'fand and G. E. Shilov, Generalized Functions, Vol.1, Academic Press, New York, 1964.

[6a] C. L. Siegel, Uber die analytische Theorie der quadratischen Formen, Ann. of Math. 36 (1935), 527-606.

[6b] - The volume of the fundamental domain for some infinite groups, Trans. Amer. Math. Soc. 39 (1936), 209-218.

[6c] — Uber die Zetafunctionen indefiniter quadratischer Formen, Math. Z. 43 (1938), 682-708.

[ 7 ] T. Ono, On Tamagawa Numbers, Proc. Sympos. Pure Math., Vol.9, Amer. Math. Soc., 1966, 122-132.

[ 8 ] A. Weil, Adeles and algebraic groups, Inst. Adv. Study, Princeton, 1961.

[9] T. Ono, A mean value theorem in adele geometry, J. Math. Soc. Japan, Vol. 20, Nos. 1-2, 1968, 275-288.

[10] - On algebraic groups and discontinuous groups, Nagoya Math. J., Vol. 27, 1966, 279-322. 
[11] I. N. Bernstein and S. I. Gel'fand, Meromorphic property of the Functions $P$, Funct. anal. and its appl. 3(1) (1969), 84-86.

[12] S. Lang and A. Weil, Number of points of varieties in finite fields, Amer. J. Math. 76 (1954).

[13] F. Sato, Eisenstein Series for Indefinite Quadratic Forms, (to appear).

[14] T. Suzuki, Fourier transforms of relative invariants of prehomogenous vector space, Master Thesis in Japanese, Nagoya Univ., 1975.

Department of Mathematics

Nagoya University 